\title{
Smoothed Performance Guarantees for Local Search *
}

\author{
Tobias Brunsch \\ Dept. of Computer Science \\ University of Bonn, Germany \\ brunsch@cs.uni-bonn.de \\ Cyriel Rutten \\ Dept. of Quantitative Economics \\ Maastricht University, The Netherlands \\ cyrielrutten@gmail.com
}

\author{
Heiko Röglin \\ Dept. of Computer Science \\ University of Bonn, Germany \\ heiko@roeglin.org \\ Tjark Vredeveld \\ Dept. of Quantitative Economics \\ Maastricht University, The Netherlands \\ t.vredeveld@maastrichtuniversity.nl
}

\begin{abstract}
We study popular local search and greedy algorithms for standard machine scheduling problems. The performance guarantee of these algorithms is well understood, but the worst-case lower bounds seem somewhat contrived and it is questionable whether they arise in practical applications. To find out how robust these bounds are, we study the algorithms in the framework of smoothed analysis, in which instances are subject to some degree of random noise.

While the lower bounds for all scheduling variants with restricted machines are rather robust, we find out that the bounds are fragile for unrestricted machines. In particular, we show that the smoothed performance guarantee of the jump and the lex-jump algorithm are (in contrast to the worst case) independent of the number of machines. They are $\Theta(\phi)$ and $\Theta(\log \phi)$, respectively, where $1 / \phi$ is a parameter measuring the magnitude of the perturbation. The latter immediately implies that also the smoothed price of anarchy is $\Theta(\log \phi)$ for routing games on parallel links. Additionally, we show that for unrestricted machines also the greedy list scheduling algorithm has an approximation guarantee of $\Theta(\log \phi)$.
\end{abstract}

\section{Introduction}

The performance guarantee of local search and greedy algorithms for scheduling problems is well studied and understood. For most algorithms, matching upper and lower bounds on their approximation ratio are known. The lower bounds are often somewhat contrived, however, and it is questionable whether they resemble typical instances in practical applications. For that reason, we study these algorithms in the framework of smoothed analysis, in which instances are subject to some degree of random noise. By doing so, we find out for which heuristics and scheduling variants the lower bounds are robust and for which they are fragile and not very likely to occur in practical applications. Since pure Nash equilibria can be seen as local optima, our results also imply a new bound on the smoothed price of anarchy, showing that known worst-case results are too pessimistic in the presence of noise.

*A preliminary version of this paper appeared in the proceedings of ESA 2011. 
Let us first describe the scheduling problems that we study. We assume that there is a set $J=\{1, \ldots, n\}$ of jobs each of which needs to be processed on one of the machines from the set $M=\{1, \ldots, m\}$. All jobs and machines are available for processing at time 0 . The goal is to schedule the jobs on the machines such that the makespan, i.e., the time at which the last job is completed, is minimized. Each machine $i \in M$ has a speed $s_{i}$ and each job $j \in J$ has a processing requirement $p_{j}$. The time $p_{i j}$ it takes to fully process job $j$ on machine $i$ depends on the machine environment. We consider two machine environments. The first one is the one of uniform parallel machines, also known as related machines: $p_{i j}=p_{j} / s_{i}$. The second machine environment that we consider is the one of restricted related machines: a job $j$ is only allowed to be processed on a subset $\mathcal{M}_{j} \subseteq M$ of the machines. The processing time is therefore $p_{i j}=p_{j} / s_{i}$ if $i \in \mathcal{M}_{j}$ and $p_{i j}=\infty$ if $i \notin \mathcal{M}_{j}$. An instance $I$ of a scheduling problem consists of the machine speeds $s_{1}, \ldots, s_{m}$, the processing requirements $p_{1}, \ldots, p_{n}$, and in the restricted case the allowed machine set $\mathcal{M}_{j} \subseteq M$ for every job $j$.

A special case for both machine environments is when all speeds are equal, i.e., $s_{i}=1$ for all $i \in M$. In this case, we say that the machines are identical. In the notation of Graham et al. [13] these problems are denoted by $Q \| C_{\max }$ and $Q\left|\mathcal{M}_{j}\right| C_{\max }$ for the related machine problems and $P|| C_{\max }$ and $P\left|\mathcal{M}_{j}\right| C_{\max }$ in case of identical machines. In these problems, makespan minimization is equivalent to minimizing the maximum machine finishing time. Once the assignment of the jobs to the machines is known, the order in which the jobs are processed is of no importance to determine the machine finishing times, as long as the jobs are processed without any idle time in between. Therefore, we assume that the jobs that are scheduled on a machine $i$ share this processor in such a way that they all finish at the same time.

Even in the case that all speeds are equal, the problems under consideration are known to be strongly NP-hard when $m$ is part of the input (see, e.g., Garey and Johnson [10]). This has motivated a lot of research in the previous decades on approximation algorithms for scheduling problems. Since some of the theoretically best approximation algorithms are rather involved, a lot of research has focused on simple heuristics like greedy algorithms and local search algorithms which are easy to implement. While greedy algorithms make reasonable ad hoc decisions to obtain a schedule, local search algorithms start with some schedule and iteratively improve the current schedule by performing some kind of local improvements until no such is possible anymore. In this article, we consider the following algorithms that can be applied to all scheduling variants that we have described above:

- List scheduling is a greedy algorithm that starts from an empty schedule and a list of jobs. Then, it repeatedly selects the next unscheduled job from the list and assigns it to the machine on which it will be completed the earliest with respect to the current partial schedule. We call any schedule that can be generated by list scheduling a list schedule.

- The jump and the lex-jump algorithms are local search algorithms that start with an arbitrary schedule and iteratively perform a local improvement step. In each improvement step, one job is reassigned (jumped) from a machine $i$ to a different machine $i^{\prime}$ where it finishes earlier. In the jump algorithm, only jobs on critical machines $i$, i.e., machines that have maximum finishing time, are considered to be 
improving. In the lex-jump algorithm, the jobs can be arbitrary. Note that a local step is lex-jump improving if and only if the sorted vector of machine finishing times decreases lexicographically, hence the term lex-jump. A schedule for which there is no jump improvement step or no lex-jump improvement step is called jump optimal or lex-jump optimal, respectively.

For each of these three algorithms, we are interested in their performance guarantees, i.e., the worst case bound on the ratio of the makespan of a schedule to be returned by the algorithm over the makespan of an optimal schedule. The final schedule returned by a local search algorithm is called a local optimum. Usually, there are multiple local optima for a given scheduling instance both for the jump and the lex-jump algorithm with varying quality. As we do not know which local optimum is found by the local search, we will always bound the quality of the worst local optimum. Since local optima for lex-jump and pure Nash equilibria are the same, see e.g. [26], this corresponds to bounding the price of anarchy in the scheduling game that is obtained if jobs are selfish agents trying to minimize their own completion time and if the makespan is considered as the welfare function. Similarly, list scheduling can produce different schedules depending on the order in which the jobs are inserted into the list. Also for list scheduling we will bound the quality of the worst schedule that can be obtained.

Notation. Consider an instance $I$ for the scheduling problem and a schedule $\sigma$ for this instance. By $J_{i}(\sigma) \subseteq J$ we denote the set of jobs assigned to machine $i$ according to $\sigma$. The processing requirement on a machine $i \in M$ is defined as $\sum_{j \in J_{i}(\sigma)} p_{j}$ and the load of a machine is defined by $L_{i}(I, \sigma)=\sum_{j \in J_{i}(\sigma)} p_{i j}$. The makespan $C_{\max }(I, \sigma)$ of $\sigma$ can be written as $C_{\max }(I, \sigma)=\max _{i \in M} L_{i}(I, \sigma)$. The optimal makespan, i.e., the makespan of an optimal schedule is denoted by $C_{\max }^{*}(I)$. By $\operatorname{Jump}(I), \operatorname{Lex}(I)$, and $\operatorname{List}(I)$ we denote the set of all feasible jump optimal schedules, lex-jump optimal schedules, and list schedules, respectively, according to instance $I$.

If the instance $I$ is clear from the context, we simply write $L_{i}(\sigma)$ instead of $L_{i}(I, \sigma)$, $C_{\max }(\sigma)$ instead of $C_{\max }(I, \sigma)$, and $C_{\max }^{*}$ instead of $C_{\max }^{*}(I)$. If the schedule $\sigma$ is clear as well, we simplify our notation further to $L_{i}$ and $C_{\max }$ and we write $J_{i}$ instead of $J_{i}(\sigma)$. By appropriate scaling, we may assume w.l.o.g. that the slowest machine has speed $s_{\min }=1$ and that all processing requirements are bounded by $p_{j} \leq 1$. In Appendix $\mathrm{A}$, the notation is summarized in a table.

Smoothed analysis. As can be seen in Table 1 , the worst-case approximation guarantee of jump and lex-jump is known for all scheduling variants and it is constant only for the simplest case with unrestricted and identical machines. In all other cases it increases with the number $m$ of machines. For list scheduling, the case with unrestricted and related machines has been considered. Cho and Sahni [6] and Aspnes et al. [2] showed that the performance guarantee of list scheduling is $\Theta(\log m)$ in this case.

In order to analyze the robustness of the worst-case bounds, we turn to the framework of smoothed analysis, introduced by Spielman and Teng [24] to explain why certain algorithms perform well in practice in spite of a poor worst-case running time. Smoothed analysis is a hybrid of average-case and worst-case analysis: First, an adversary chooses 
an instance. Second, this instance is slightly randomly perturbed. The smoothed performance is the expected performance, where the expectation is taken over the random perturbation. The adversary, trying to make the algorithm perform as bad as possible, chooses an instance that maximizes this expected performance. This assumption is made to model that often the input an algorithm gets is subject to imprecise measurements, rounding errors, or numerical imprecision. If the smoothed performance guarantee of an algorithm is small, then bad worst-case instances might exist, but one is very unlikely to encounter them if instances are subject to some small amount of random noise.

We follow the more general model of smoothed analysis introduced by Beier and Vöcking [5]. In this model, the adversary is even allowed to specify the probability distribution of the random noise. The influence he can exert is described by a parameter $\phi \geq 1$ denoting the maximum density of the noise. This model is formally defined as follows.

Definition 1. In a $\phi$-smooth instance $\mathcal{I}$, the adversary chooses the following input data:

- the number $m$ of machines;

- arbitrary machine speeds $s_{\max }:=s_{1} \geq \ldots \geq s_{m}=: s_{\min }=1$, in the case of nonidentical machines;

- the number $n$ of jobs;

- an arbitrary set $\mathcal{M}_{j} \subseteq M$ for each job $j \in J$, in the case of restricted machines;

- for each $p_{j}$, a probability density $f_{j}:[0,1] \rightarrow[0, \phi]$ according to which $p_{j}$ is chosen independently of the processing requirements of the other jobs.

Note that the only perturbed part of the instance are the processing requirements. Formally, a $\phi$-smooth instance is not a single instance but a distribution over instances. We write $I \sim \mathcal{I}$ to denote that the instance $I$ is drawn from the $\phi$-smooth instance $\mathcal{I}$.

The parameter $\phi$ specifies how close the analysis is to a worst case analysis. The adversary can, for example, choose for every $p_{j}$ an interval of length $1 / \phi$ from which $p_{j}$ is drawn uniformly at random. For $\phi=1$, every processing requirement is uniformly distributed over $[0,1]$, and hence the input model equals the average case for uniformly distributed processing times. When $\phi$ gets larger, the adversary can specify the processing requirements more and more precisely, and for $\phi \rightarrow \infty$ the smoothed analysis approaches a worst-case analysis.

In this article, we analyze the smoothed performance guarantee of the jump, the lexjump, and the list scheduling algorithm. As mentioned above, to define the approximation guarantee of these algorithms on a given instance, we consider the worst local optimum (for the jump and the lex-jump algorithm) or the worst order in which the jobs are inserted into the list (for the list scheduling algorithm). Now, the smoothed performance is defined to be the worst expected approximation guarantee of any $\phi$-smooth instance.

Our results. Our results for the jump and lex-jump algorithm are summarized in Table 1. The first remarkable observation is that the smoothed performance guarantees for all variants of restricted machines are robust against random noise. We show that even 


\begin{tabular}{|c|c|c|c|c|c|c|}
\hline & \multicolumn{2}{|c|}{ worst case } & \multicolumn{4}{|c|}{$\phi$-smooth } \\
\hline & jump & lex-jump & jump & & lex-jump & \\
\hline $\begin{array}{l}\text { unrestricted } \\
\text { identical }\end{array}$ & $\Theta(1), 9,23$ & $\Theta(1), 9,23$ & $\Theta(1)$ & & $\Theta(1)$ & \\
\hline $\begin{array}{l}\text { unrestricted } \\
\text { related }\end{array}$ & $\Theta(\sqrt{m}), 623$ & $\Theta\left(\frac{\log m}{\log \log m}\right)$ & $\Theta(\phi)[2.1]$ & & $\Theta(\log \phi)] 2.2$ & 2.3 \\
\hline $\begin{array}{l}\text { restricted } \\
\text { identical }\end{array}$ & $\Theta(\sqrt{m})[21$ & $\Theta\left(\frac{\log m}{\log \log m}\right) 3$ & $\Theta(\sqrt{m}) 3.1$ & & $\Theta\left(\frac{\log m}{\log \log m}\right)$ & 3.2 \\
\hline $\begin{array}{l}\text { restricted } \\
\text { related }\end{array}$ & $\Theta\left(\sqrt{m \cdot s_{\max }}\right) 21$ & $\Theta\left(\frac{\log S}{\log \log S}\right) 21$ & $\Theta\left(\sqrt{m \cdot s_{\max }}\right)$ & 3.1 & $\Omega\left(\frac{\log m}{\log \log m}\right)$ & 3.2 \\
\hline
\end{tabular}

Table 1: Worst-case and smoothed performance guarantees for jump and lex-jump optimal schedules. Here, $S=\sum_{i=1}^{m} s_{i}$, and we assume w.l.og. that $s_{\min }=1$. With [X.Y] we refer to the section in this article where the bound is shown.

for large perturbations with constant $\phi$, the worst-case lower bounds carry over. This can be seen as an indication that neither the jump algorithm nor the lex-jump algorithm yield a good approximation ratio for scheduling with restricted machines in practice.

The situation is much more promising for the unrestricted variants. Here, the worstcase bounds are fragile and do not carry over to the smoothed case. The interesting case is the one of unrestricted and related machines. Even though both for jump and for lexjump the worst-case lower bound is not robust, there is a significant difference between these two: while the smoothed approximation ratio for jump grows linearly with the perturbation parameter $\phi$, it grows only logarithmically in $\phi$ for lex-jump optimal schedules. This proves that also in the presence of random noise lex-jump optimal schedules are significantly better than jump optimal schedules. As mentioned earlier, this also implies that the smoothed price of anarchy is $\Theta(\log \phi)$. Additionally, we show that the smoothed approximation ratio of list scheduling is $\Theta(\log \phi)$ as well, even when the order of the list may be specified after the realizations of the processing times are known. This indicates that both the lex-jump algorithm and the list scheduling algorithm should yield good approximations on practical instances.

Related work. The approximability of $Q \| C_{\max }$ is well understood. Cho and Sahni [6] showed that list scheduling has a performance guarantee of at most $1+\sqrt{2 m-2} / 2$ for $m \geq 3$ and that it is at least $\Omega(\log m)$. Aspnes et al. 2] improved the upper bound to $O(\log m)$ matching the lower bound asymptotically. Hochbaum and Shmoys [14] designed a polynomial time approximation scheme for this problem. Polynomial time approximation algorithms and polynomial time approximation schemes for special cases of the problem on restricted related machines are given in, among others, [18, 11, 20]. More work on restricted related parallel machines is discussed in the survey of Leung and $\mathrm{Li}$ [17].

In the last decade, there has been a strong interest in understanding the worst-case behavior of local optima. We refer to the survey [1] and the book [19] for a comprehensive overview of the worst-case analysis and other theoretical aspects of local search. It follows from the work of Cho and Sahni [6] that for the problem on unrestricted related machines the performance guarantee of the jump algorithm is $(1+\sqrt{4 m-3}) / 2$ and this bound is tight [23]. For lex-jump optimal schedules, Czumaj and Vöcking [7] showed that the performance guarantee is $\Theta\left(\min \left\{\frac{\log m}{\log \log m}, \log s_{\max }\right\}\right)$. For the problem on restricted related 
machines, Rutten et al. 21] showed that the performance guarantee of locally optimal schedules with respect to the jump neighborhood is $\left(1+\sqrt{1+4(m-1) s_{\max }}\right) / 2$ and that this bound is tight up to a constant factor. Moreover, they showed that the performance guarantee of lex-jump optimal schedules is $\Theta\left(\frac{\log S}{\log \log S}\right)$, where $S=\sum_{i=1}^{m} s_{i}$. When all speeds are equal, Awerbuch et al. [3] showed that the performance guarantee for lex-jump optimal schedules is $\Theta\left(\frac{\log m}{\log \log m}\right)$.

Up to now, smoothed analysis has been mainly applied to running time analysis (see, e.g., 25] for a survey). The first exception is the paper by Becchetti et al. [4 who introduced the concept of smoothed competitive analysis, which is equivalent to smoothed performance guarantees for online algorithms. Schäfer and Sivadasan [22] performed a smoothed competitive analysis for metrical task systems. Englert et al. [8] considered the 2-Opt algorithm for the traveling salesman problem and determined, among others, the smoothed performance guarantee of local optima of the 2-Opt algorithm. Hoefer and Souza [15] presented one of the first average case analyses for the price of anarchy.

The remainder of this article is organized as follows. In Section 2, we provide asymptotically matching upper and lower bounds on the smoothed performance guarantees of jump optimal, lex-jump optimal, and list schedules in case of unrestricted related machines. In Section 3, we show that smoothing does not help for the setting of restricted machines.

\section{Unrestricted Related Machines}

\section{$2.1 \quad$ Jump Optimal Schedules}

We show that the smoothed performance guarantee grows linearly with the smoothing parameter $\phi$ and is independent of the number of jobs and machines. In particular, it is constant if the smoothing parameter is constant. In proving our results, we make use of the following proposition which follows from Cho and Sahni [6].

Proposition 2. For any scheduling instance $I$ with $m$ unrestricted related machines and $n$ jobs

$$
\max _{\sigma \in \operatorname{Jump}(I)} \frac{C_{\max }(I, \sigma)}{C_{\max }^{*}(I)} \leq \frac{1+\sqrt{4 \min \{m, n\}-3}}{2} \leq \frac{1}{2}+\sqrt{n} .
$$

Theorem 3. For any $\phi$-smooth instance $\mathcal{I}$ with unrestricted and related machines,

$$
\underset{I \sim \mathcal{I}}{\mathbf{E}}\left[\max _{\sigma \in \mathrm{Jump}(I)} \frac{C_{\max }(I, \sigma)}{C_{\max }^{*}(I)}\right]<5.1 \phi+2.5=O(\phi) .
$$

Proof. First note that if $m>n$, then there exist an optimal schedule and a worst jumpoptimal schedule that do not schedule any job on any of the slowest $m-n$ machines. We ignore these slowest $m-n$ machines, and therefore we assume that $m \leq n$. We will prove an upper bound on the performance guarantee of jump optimal schedules that decreases when the sum of processing requirements $Q=\sum_{j \in J} p_{j}$ increases and that is valid for 
every instance. Then, we will argue that for $\phi$-smooth instances $Q$ is usually not too small, which yields the theorem.

Let $\sigma$ denote an arbitrary jump optimal schedule for some arbitrary processing requirements $p_{j} \in[0,1]$. Let $i$ be an arbitrary machine, let machine $i_{\max }$ be a critical machine in schedule $\sigma$, and let $j$ be a job assigned to machine $i_{\max }$ by schedule $\sigma$. By jump optimality of $\sigma$ it follows that

$$
C_{\max }(\sigma)=L_{i_{\max }} \leq L_{i}+p_{j} / s_{i} \leq L_{i}+p_{\max } / s_{i},
$$

where $p_{\max }$ denotes the processing requirement of the largest job. The previous inequality yields that $s_{i} \cdot C_{\max }(\sigma) \leq s_{i} \cdot L_{i}+p_{\max }$ for all machines $i \in M$. Summing over all machines from $M \backslash\left\{i_{\max }\right\}$ and adding $s_{i_{\max }} \cdot L_{i_{\max }}$ to both sides of the inequality, we find that

$$
\sum_{i \in M} s_{i} \cdot C_{\max }(\sigma) \leq \sum_{i \in M \backslash\left\{i_{\max }\right\}} p_{\max }+\sum_{i \in M} s_{i} \cdot L_{i} \leq(n-1) \cdot p_{\max }+\sum_{i \in M} s_{i} \cdot L_{i}
$$

since $L_{i_{\max }}=C_{\max }(\sigma)$. Noting that $\sum_{i \in M} s_{i} \cdot L_{i}=\sum_{j \in J} p_{j}=Q$ yields the following upper bound on the makespan of any jump optimal schedule $\sigma$ :

$$
C_{\max }(\sigma) \leq \frac{Q}{\sum_{i \in M} s_{i}}+\frac{n-1}{\sum_{i \in M} s_{i}},
$$

where the last inequality follows since $p_{\max } \leq 1$. Using the well-known bound $C_{\max }^{*} \geq$ $Q / \sum_{i \in M} s_{i}$ we obtain

$$
C_{\max }(\sigma) \leq \frac{Q}{\sum_{i \in M} s_{i}}+\frac{n-1}{\sum_{i \in M} s_{i}} \leq\left(1+\frac{n-1}{Q}\right) \cdot C_{\max }^{*} .
$$

Hence,

$$
\max _{\sigma \in \operatorname{Jump}(I)} \frac{C_{\max }(I, \sigma)}{C_{\max }^{*}(I)} \leq 1+\frac{n-1}{Q} .
$$

The performance guarantee of any jump optimal schedule can only be bad if $Q$ is small. Since the instance is $\phi$-smooth, the processing requirements are random variables in $[0,1]$ with bounded densities. Let $\mathcal{F}$ denote the failure event that $Q \leq(n-\sqrt{n \ln n}) /(2 \phi)$. We define $x_{j}$ to be independent random variables drawn uniformly from $[0,1 / \phi]$ for all $j \in J$. Then, $\operatorname{Pr}\left[p_{j} \geq a\right] \geq \operatorname{Pr}\left[x_{j} \geq a\right]$ for any $a \in[0,1]$. Let $X=\sum_{j \in J} x_{j}$. Then, for any $a \in[0, n]$, it follows that $\operatorname{Pr}[Q \geq a] \geq \operatorname{Pr}[X \geq a]$. Hence,

$$
\begin{aligned}
\operatorname{Pr}[\mathcal{F}] & =\operatorname{Pr}\left[Q \leq \frac{n-\sqrt{n \ln n}}{2 \phi}\right] \leq \operatorname{Pr}\left[X \leq \frac{n-\sqrt{n \ln n}}{2 \phi}\right] \\
& =\operatorname{Pr}\left[\mathbf{E}[X]-X \geq \frac{\sqrt{n \ln n}}{2 \phi}\right] \leq e^{-(\ln n) / 2}=\frac{1}{\sqrt{n}},
\end{aligned}
$$

where the last inequality follows from Hoeffding's bound [16] (see also Theorem 31 in the appendix). Consider the random variable

$$
Z= \begin{cases}\frac{1}{2}+\sqrt{n} & \text { if event } \mathcal{F} \text { occurs } \\ 1+\frac{n-1}{Q} & \text { otherwise }\end{cases}
$$


and let $Y=\max _{\sigma \in \operatorname{Jump}(I)} \frac{C_{\max }(I, \sigma)}{C_{\max }^{*}(I)}$. Due to Inequalities (1) and (2) we have $Y \leq Z$. We denote by $\overline{\mathcal{F}}$ the complement of $\mathcal{F}$ and obtain

$$
\begin{aligned}
\underset{I \sim \mathcal{I}}{\mathbf{E}}[Y] & \leq \underset{I \sim \mathcal{I}}{\mathbf{E}}[Z] \leq \underset{I \sim \mathcal{I}}{\mathbf{E}}[Z \mid \overline{\mathcal{F}}]+\underset{I \sim \mathcal{I}}{\mathbf{E}}[Z \mid \mathcal{F}] \cdot \underset{I \sim \mathcal{I}}{\operatorname{Pr}}[\mathcal{F}] \\
& \stackrel{\text { 3in }}{\leq}\left(1+\frac{2 \phi(n-1)}{n-\sqrt{n \ln n}}\right)+\frac{1 / 2+\sqrt{n}}{\sqrt{n}} \\
& <2.5+\frac{2 \phi}{1-\sqrt{\ln (n) / n}}<2.5+5.1 \phi .
\end{aligned}
$$

For the third inequality, we used $Q>(n-\sqrt{n \ln n}) /(2 \phi)$ if event $\mathcal{F}$ does not hold. The last inequality holds since

$$
\max _{n \in \mathbb{Z}^{+}} \frac{2}{1-\sqrt{\ln (n) / n}}<5.1,
$$

where the maximum is attained for $n=3$.

Corollary 4. Consider an instance of scheduling with unrestricted and related machines in which the processing requirement of every job is chosen independently and uniformly at random from $[0,1]$. The expected performance guarantee of the worst jump optimal schedule is $O(1)$.

Next, we show that the upper bound on the smoothed performance guarantee provided in Theorem 3 is tight up to constant factor when $\phi \geq 2$.

Theorem 5. There is a class of $\phi$-smooth instances $\mathcal{I}$ with unrestricted and related machines such that

$$
\underset{I \sim \mathcal{I}}{\mathbf{E}}\left[\max _{\sigma \in \mathrm{Jump}(I)} \frac{C_{\max }(I, \sigma)}{C_{\max }^{*}(I)}\right]=\Omega(\phi) .
$$

Proof. For any $\phi>2$ we construct a $\phi$-smooth instance $\mathcal{I}$ with $n=\left\lceil 4 \phi^{2}+1\right\rceil$ and $m=n$ machines. Let

$$
s_{1}=\frac{n-1}{4 \phi} \geq \phi>2 \quad \text { and } \quad s_{2}=\ldots=s_{n}=1 .
$$

We assume that the processing requirement $p_{1}$ is chosen uniformly from the interval $11-$ $1 / \phi, 1]$ while the processing requirements of all other jobs are chosen uniformly from the interval $[0,1 / \phi]$. In an optimal schedule, job 1 is scheduled on machine 1 , and all other machines process exactly one job (see Figure 1). Hence,

$$
C_{\max }^{*}=\max \left\{\frac{p_{1}}{s_{1}}, p_{2}, \ldots, p_{n}\right\} \leq \max \left\{\frac{1}{s_{1}}, \frac{1}{\phi}\right\}=\frac{1}{\phi} .
$$

We show that with high probability there exists a jump optimal schedule $\sigma$ with $C_{\max }(\sigma)>$ $1-1 / \phi$. In order to find such a schedule $\sigma$, we first schedule job 1 on machine 2 . Then, we consider the remaining jobs one after another and schedule unassigned jobs to machine 1 


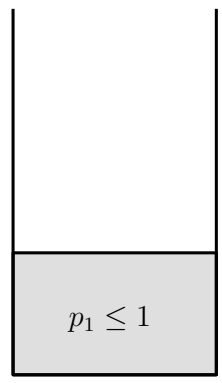

$s_{1} \approx \phi$

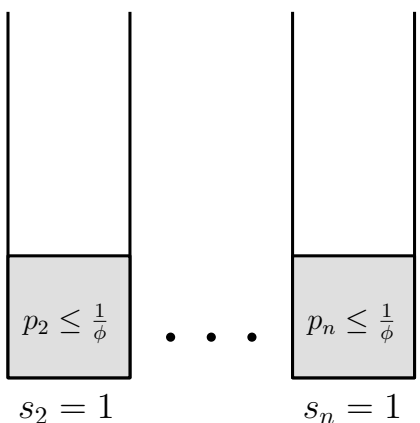

$s_{2}=1$ $s_{n}=1$
Figure 1: Optimal schedule

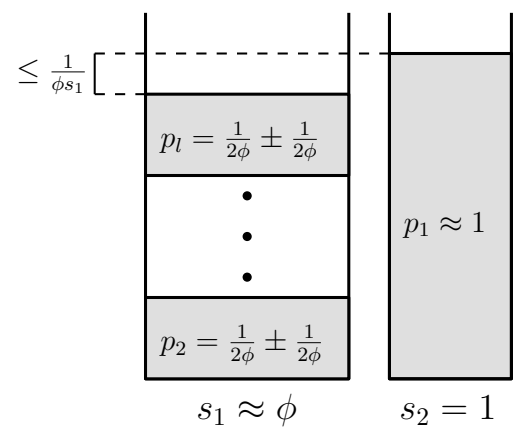

Figure 2: Machines 1 and 2 of schedule $\sigma$ if event $\mathcal{E}$ occurs

until either $L_{1} \in\left[L_{2}-1 /\left(\phi s_{1}\right), L_{2}\right)$ or all jobs are scheduled. Any job that remains unscheduled is then exclusively assigned to one empty machine. Let $\mathcal{E}$ denote the event that $Q_{2}:=\sum_{j=2}^{n} p_{j} \geq s_{1}$. Note that $\mathbf{E}\left[Q_{2}\right]=(n-1) /(2 \phi)=2 s_{1}$. We will see that event $\mathcal{E}$ holds with high probability with respect to $\phi$.

Consider the case that event $\mathcal{E}$ occurs. Then, schedule $\sigma$ is such that $L_{1} \in\left[L_{2}-\right.$ $\left.1 /\left(\phi s_{1}\right), L_{2}\right)$ since $Q_{2} / s_{1} \geq 1 \geq p_{1}=L_{2}$ and $p_{j} \leq 1 / \phi$ for all jobs $j=2, \ldots, n$ (see Figure 2). Now, we argue that schedule $\sigma$ is jump optimal. First observe that machine 2 defines the makespan since $L_{2}>\max \left\{L_{1}, p_{2} / 1, \ldots, p_{n} / 1\right\}$. Job 1 , which is the only job assigned to that machine, cannot jump to a machine $i>2$ because these have the same speed as machine 2. Furthermore, it cannot jump to machine 1 because

$$
L_{1}+\frac{p_{1}}{s_{1}} \geq L_{2}-\frac{1}{\phi s_{1}}+\frac{1-1 / \phi}{s_{1}}=L_{2}+\frac{1-2 / \phi}{s_{1}}>L_{2}
$$

as $\phi>2$. Hence, $\sigma$ is a jump optimal schedule with

$$
\frac{C_{\max }(\sigma)}{C_{\max }^{*}}>\frac{1-1 / \phi}{1 / \phi}=\phi-1 .
$$

It remains to determine the probability of event $\mathcal{E}$. Recalling $\mathbf{E}\left[Q_{2}\right]=2 s_{1}, s_{1}=(n-$ 1) $/(4 \phi)$, and $n \geq 4 \phi^{2}+1$, this can be bounded with Hoeffding's bound [16] (see also Theorem 31) as follows:

$$
\begin{aligned}
\operatorname{Pr}[\overline{\mathcal{E}}] & =\operatorname{Pr}\left[Q_{2}<s_{1}\right]=\operatorname{Pr}\left[\mathbf{E}\left[Q_{2}\right]-Q_{2}>s_{1}\right] \leq \exp \left(\frac{-2 s_{1}^{2}}{(n-1) / \phi^{2}}\right) \\
& =\exp \left(\frac{-2\left(\frac{n-1}{4 \phi}\right)^{2}}{(n-1) / \phi^{2}}\right)=\exp \left(-\frac{n-1}{8}\right) \leq \exp \left(-\frac{\phi^{2}}{2}\right) .
\end{aligned}
$$

Let $X=\max _{\sigma \in \operatorname{Jump}(I)} \frac{C_{\max }(I, \sigma)}{C_{\max }^{*}(I)}$. Applying Inequality (4) the smoothed performance guarantee can be bounded from below as follows:

$$
\underset{I \sim \mathcal{I}}{\mathbf{E}}[X] \geq \underset{I \sim \mathcal{I}}{\mathbf{E}}[X \mid \mathcal{E}] \cdot \underset{I \sim \mathcal{I}}{\operatorname{Pr}}[\mathcal{E}] \geq(\phi-1) \cdot\left(1-\exp \left(-\frac{\phi^{2}}{2}\right)\right)
$$




$$
=(\phi-1)-(\phi-1) \cdot \exp \left(-\frac{\phi^{2}}{2}\right)>\phi-1.14=\Omega(\phi),
$$

where the last inequality follows because $(\phi-1) \cdot \exp \left(-\phi^{2} / 2\right)<0.14$ for $\phi>2$.

\subsection{Upper Bounds for List Schedules and Lex-jump Optimal Schedules}

Although the worst case performance bound on unrestricted related machines for list scheduling is slightly worse than the one for lex-jump scheduling, we show that the smoothed performance guarantee of both schedules is $O(\log \phi)$. In the next subsection, we show that this bound is asymptotically tight.

Theorem 6. Let $\alpha$ be an arbitrary positive real. For $\phi \geq 2$ and any $\phi$-smooth instance $\mathcal{I}$ with unrestricted and related machines

$$
\underset{I \sim \mathcal{I}}{\operatorname{Pr}}\left[\max _{\sigma \in \operatorname{Lex}(I) \cup \operatorname{List}(I)} \frac{C_{\max }(I, \sigma)}{C_{\max }^{*}(I)} \geq \alpha\right] \leq\left(\frac{32 \phi}{2^{\alpha / 6}}\right)^{n / 2}
$$

and

$$
\underset{I \sim \mathcal{I}}{\mathbf{E}}\left[\max _{\sigma \in \operatorname{Lex}(I) \cup \operatorname{List}(I)} \frac{C_{\max }(I, \sigma)}{C_{\max }^{*}(I)}\right] \leq 18 \log _{2} \phi+30=O(\log \phi) .
$$

Note that the assumption $\phi \geq 2$ in Theorem 6 is no real restriction as for $\phi \in[1,2)$ any $\phi$-smooth instance is a 2 -smooth instance. Hence, for these values we can apply all bounds from Theorem 6 when substituting $\phi$ by 2 . In particular, the expected value is a constant.

In the remainder of this section, we will use the following notation (see also Appendix A . Let $J_{i, j}(\sigma)$ denote the set of all jobs that are scheduled on machine $i$ and have index at most $j$, i.e., $J_{i, j}(\sigma)=J_{i}(\sigma) \cap\{1, \ldots, j\}$. If $\sigma$ is clear from the context, then we just write $J_{i, j}$. We start with observing an essential property that both lex-jump optimal schedules and list schedules have in common.

Definition 7. We call a schedule $\sigma$ on machines $1, \ldots, m$ with speeds $s_{1}, \ldots, s_{m}$ a near list schedule, if we can index the jobs in such a way that

$$
L_{i^{\prime}}+\frac{p_{j}}{s_{i^{\prime}}} \geq L_{i}-\sum_{\ell \in J_{i, j-1}(\sigma)} \frac{p_{\ell}}{s_{i}}
$$

for all machines $i^{\prime} \neq i$ and all jobs $j \in J_{i}(\sigma)$. With $\mathrm{NL}(I)$ we denote the set of all near list schedules for instance $I$.

Inequality (5) can be interpreted as follows. Assume that the jobs are already indexed correctly and imagine that on each machine the jobs form a stack, ordered from top to bottom ascendingly according to their index. Now, consider an arbitrary job $j$ on machine $i$ (see Figure 3a). Inequality (5) states that the completion time of job $j$ after removing all jobs above $j$ is minimized on machine $i$ in case only job $j$ is allowed to move (see Figure 3b.

Lemma 8. For any instance $I$ the relation $\operatorname{Lex}(I) \cup \operatorname{List}(I) \subseteq \mathrm{NL}(I)$ holds. 


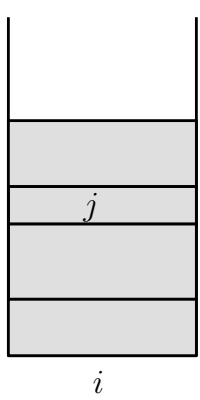

(a) Jobs on machine $i$, including job $j$, visualized as a stack

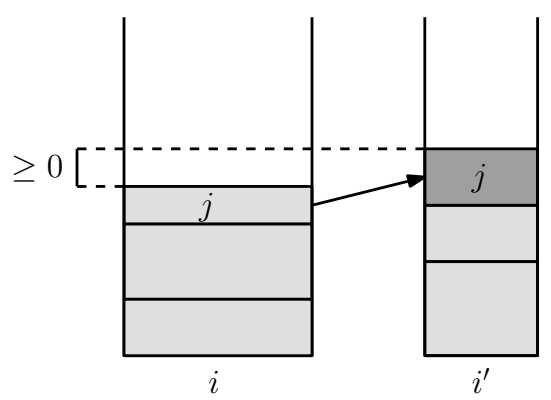

(b) Job $j$ does not benefit from jumping to machine $i^{\prime}$

Figure 3: Interpretation of Inequality (5)

Note that in general neither $\operatorname{Lex}(I) \subseteq \operatorname{List}(I)$ nor List $(I) \subseteq \operatorname{Lex}(I)$ holds (see Figure 4). Moreover, there also exist near list schedules that are neither in $\operatorname{Lex}(I)$ nor in $\operatorname{List}(I)$ (see Figure 4c), i.e., near list schedules are a non-trivial generalization of both lex-jump optimal schedules and list schedules.

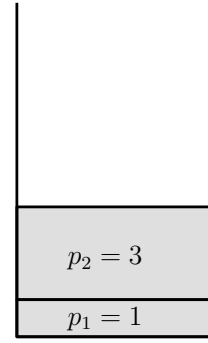

$s_{1}=3$

(a) A list schedule wh not lex-jump optimal

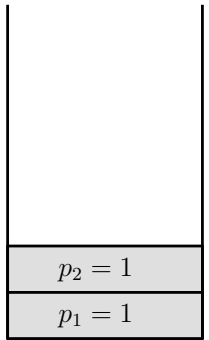

$s_{1}=7$ (b) A lex-jump optimal schedule which is no list schedule

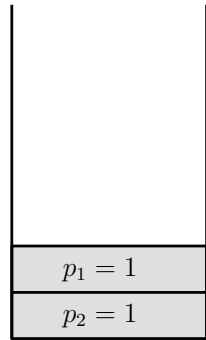

$s_{1}=7$

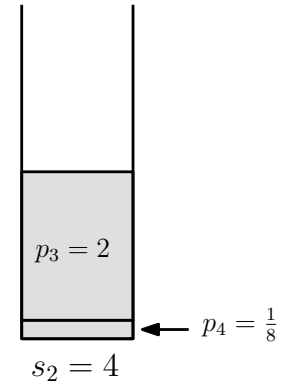

(c) A near list schedule which is neither lex-jump optimal nor a list schedule

Figure 4: Relationship between $\operatorname{Lex}(I), \operatorname{List}(I)$, and $\mathrm{NL}(I)$

Proof of Lemma 8. For any schedule $\sigma \in \operatorname{Lex}(I)$, we can index the jobs arbitrarily and, by definition, even the stronger inequality $L_{i^{\prime}}+p_{j} / s_{i^{\prime}} \geq L_{i}$ holds. For $\sigma \in \operatorname{List}(I)$ we can index the jobs in reverse order in which they appear in the list that was used for list scheduling. Consider an arbitrary job $j \in J_{i}(\sigma)$ and a machine $i^{\prime} \neq i$. Let $L_{i}^{\prime}, L_{i^{\prime}}^{\prime}$ and $L_{i}, L_{i^{\prime}}$ denote the loads of machines $i$ and $i^{\prime}$ before assigning job $j$ to machine $i$ and the loads of $i$ and $i^{\prime}$ in the final schedule, respectively. Then, $L_{i}^{\prime}+p_{j} / s_{i} \leq L_{i^{\prime}}^{\prime}+p_{j} / s_{i^{\prime}}$ as $j$ is assigned to machine $i$ according to list scheduling. Since $L_{i}=L_{i}^{\prime}+\sum_{\ell \in J_{i, j}} p_{\ell} / s_{i}$ and $L_{i^{\prime}} \geq L_{i^{\prime}}^{\prime}$, this implies $L_{i^{\prime}}+p_{j} / s_{i^{\prime}} \geq L_{i^{\prime}}^{\prime}+p_{j} / s_{i^{\prime}} \geq L_{i}^{\prime}+p_{j} / s_{i}=L_{i}-\sum_{\ell \in J_{i, j-1}} p_{\ell} / s_{i}$.

In the remainder, we fix an instance $I$ and consider an arbitrary schedule $\sigma \in \operatorname{NL}(I)$ 
with appropriate indices of the jobs such that Inequality $(5)$ holds. To prove Theorem 6 . we show that in case the ratio of $C_{\max }(I, \sigma)$ over $C_{\max }^{*}(I)$ is large, then instance $I$ needs to have many very small jobs, see Corollary 17 . This holds even when the instance $I$ is deterministically picked by some adversary. This observation allows us to prove the main theorem of this subsection by showing that for any $\phi$-smooth instance, there are only "few" small jobs in expectation. The latter implies that a large ratio only happens with (exponentially) small probability.

In our proofs, we adopt some of the notation also used by Czumaj and Vöcking 7 (see also Appendix A). Given a schedule $\sigma$, we set $c=\left\lfloor C_{\max }(\sigma) / C_{\max }^{*}\right\rfloor-1$. Recall that the machines are ordered such that $s_{1} \geq \ldots \geq s_{m}$. For any integer $k \leq c$ let $H_{k}=\left\{1, \ldots, i_{k}\right\}$ where $i_{k}=\max \left\{i \in M: L_{i^{\prime}} \geq k \cdot C_{\max }^{*} \forall i^{\prime} \leq i\right\}$. Note that $i_{k}=m$ for all $k \leq 0$ and hence $H_{k}=M$ for such $k$ (see Figure 5). Further, define $R_{k}=H_{k} \backslash H_{k+1}$ for all $k \in\{0, \ldots, c-1\}$ and $R_{c}=H_{c}$. Note that this classification always refers to schedule $\sigma$ even if additionally other schedules are considered. Some properties follow straightforwardly.

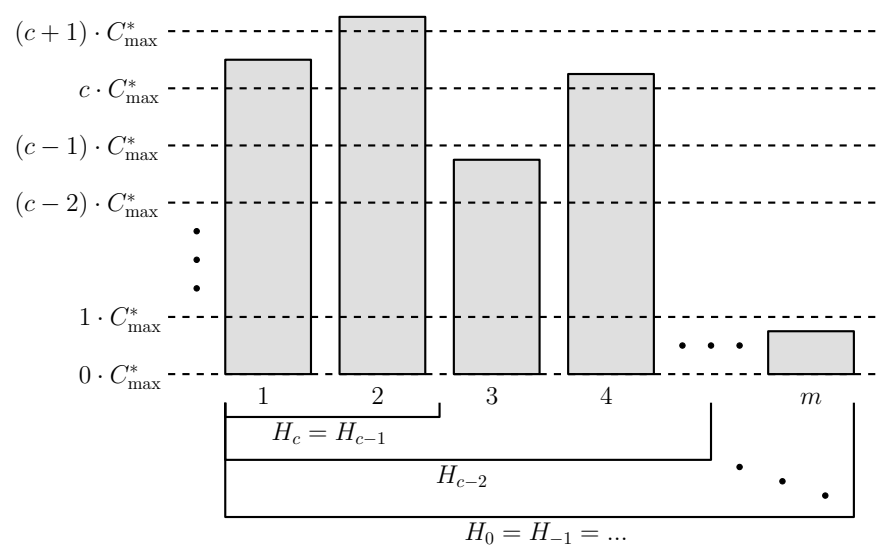

Figure 5: Machine classification by Czumaj and Vöcking

Property 1. For each machine $i \in H_{k}, L_{i} \geq k \cdot C_{\max }^{*}$.

Property 2. Machine $i_{k}+1$, if it exists, is the first machine in $M \backslash H_{k}$, i.e., the machine with the least index, and, hence, a fastest machine in $M \backslash H_{k}$.

Property 3. $L_{i_{k}+1}<k \cdot C_{\max }^{*}$ for all $k \in\{1, \ldots, c\}$, and $L_{1}<(c+2) \cdot C_{\max }^{*}$.

As mentioned, we need to show that there are many small jobs. To do so, we will show that the the speeds of the machines in low classes, i.e., $R_{0}$ and $R_{1}$, are exponentially small with respect to the machines in the highest class $R_{c}$ (Lemma 14) and that the machines in low classes need to process high volume (Lemma 13). We start by showing that the highest class is nonempty.

Lemma 9. Machine 1 is in class $R_{c}$.

Proof. Let $i$ be a critical machine. If $i=1$, then we obtain $L_{1} / C_{\max }^{*}=C_{\max }(\sigma) / C_{\max }^{*}>c$. Otherwise we apply Inequality (5) for the job $j=\min \left\{\ell \in J_{i}\right\}$ with the smallest index 
on machine $i$ and for machine 1 . This yields $L_{1}+p_{j} / s_{1} \geq L_{i}$. Hence, $L_{1} / C_{\max }^{*} \geq$ $L_{i} / C_{\max }^{*}-\left(p_{j} / s_{1}\right) / C_{\max }^{*} \geq C_{\max }(\sigma) / C_{\max }^{*}-1 \geq c$, where the second inequality is due to the fact that any job can contribute at most $C_{\max }^{*}$ to the makespan of a fastest machine.

Let $t$ and $k$ be integers satisfying $0 \leq t \leq k \leq c$. Several times we will consider the first many jobs on some machine $i \in H_{k}$ which contribute at least $t \cdot C_{\max }^{*}$ to the load of machine $i$. We denote the set of those jobs by $J_{i, \geq t}$. Formally,

$$
J_{i, \geq t}=J_{i, j_{i}^{t}} \text { for } j_{i}^{t}=\min \left\{j: \sum_{\ell \in J_{i, j}} p_{\ell} / s_{i} \geq t \cdot C_{\max }^{*}\right\} .
$$

Using this notation, Lemma 10 and Corollary 11 restrict the machines on which a job in $J_{i, \geq t}$ can be scheduled in an optimal schedule.

Lemma 10. Let $k_{1}>k_{2}$ and $t \leq k_{1}$ be positive integers, let $i_{1} \in H_{k_{1}}$ and $i_{2} \in M \backslash H_{k_{2}}$ be machines in $H_{k_{1}}$ and not in $H_{k_{2}}$, respectively, and let $j \in J_{i_{1}, \geq t}$ be a job on machine $i_{1}$. Then, the load job $j$ would contribute to machine $i_{2}$ is bounded from below by $p_{j} / s_{i_{2}}>$ $\left(k_{1}-k_{2}-t\right) \cdot C_{\max }^{*}$.

Proof. We apply Inequality (5) for machine $i_{1}$, for the first machine $i_{2}^{\prime}$ that does not belong to $H_{k_{2}}$, and for job $j$ to obtain

$$
L_{i_{2}^{\prime}}+\frac{p_{j}}{s_{i_{2}^{\prime}}} \geq L_{i_{1}}-\sum_{\ell \in J_{i_{1}, j-1}} \frac{p_{\ell}}{s_{i_{1}}}
$$

which implies

$$
\frac{p_{j}}{s_{i_{2}^{\prime}}} \geq L_{i_{1}}-L_{i_{2}^{\prime}}-\sum_{\ell \in J_{i_{1}, j-1}} \frac{p_{\ell}}{s_{i_{1}}} .
$$

By the choice of the machines $i_{1}$ and $i_{2}^{\prime}$ and Properties 1 and 3 we obtain $L_{i_{1}} \geq k_{1} \cdot C_{\max }^{*}$ and $L_{i_{2}^{\prime}}<k_{2} \cdot C_{\max }^{*}$. Furthermore, $j \in J_{i_{1}, \geq t}$ yields $\sum_{\ell \in J_{i_{1}, j-1}} p_{\ell} / s_{i_{1}}<t \cdot C_{\max }^{*}$. Hence, $p_{j} / s_{i_{2}^{\prime}}>\left(k_{1}-k_{2}-t\right) \cdot C_{\max }^{*}$. The claim follows since $s_{i_{2}^{\prime}} \geq s_{i_{2}}$.

Corollary 11. Let $i \in H_{k}$ be an arbitrary machine and let $t \in\{1, \ldots, k\}$ be an integer. Then, in any optimal schedule any job $j \in J_{i, \geq t}$ is assigned to machines from $H_{k-t-1}$.

Proof. Assume, for contradiction, that there is a job $j \in J_{i, \geq t}$ which is assigned to a machine $i^{\prime} \in M \backslash H_{k-t-1}$ by an optimal schedule. By Lemma 10 this job causes a load of more than $(k-(k-t-1)-t) \cdot C_{\max }^{*}=C_{\max }^{*}$ on this machine contradicting the assumption that the considered schedule is optimal.

Czumaj and Vöcking [7] showed that in a lex-jump optimal schedule the speeds of any two machines which are at least two classes apart differ by a factor of at least 2. Aspnes et al. 2] showed a similar property. In general, near list schedules have a slightly weaker property.

Lemma 12. Let $k \in\{5, \ldots, c\}$ and assume $H_{k} \neq \emptyset$. The speed of any machine in class $H_{k}$ is at least twice the speed of any machine in $M \backslash H_{k-4}$. 
Proof. We may assume that $M \backslash H_{k-4} \neq \emptyset$, since otherwise all machines have a load larger than $C_{\max }^{*}$ as $H_{k} \neq \emptyset$. Let $i_{0} \in H_{k}$ and $i_{2} \in M \backslash H_{k-4}$ be arbitrary machines and consider the jobs from $\bigcup_{i \in H_{k}} J_{i, \geq 2}$. If we would assign only these jobs to machines in $H_{k}$, then there would be a machine with load at least $2 \cdot C_{\max }^{*}$. Consequently, in an optimal schedule at least one job in $\bigcup_{i^{\prime} \in H_{k}} J_{i^{\prime}, \geq 2}$ is assigned to some machine $i^{*} \in M \backslash H_{k}$, say job $j \in J_{i_{1}, \geq 2}$. Since job $j$ contributes at most $C_{\max }^{*}$ to the load of machine $i^{*}$ in this optimal schedule, this implies $p_{j} / s_{i^{*}} \leq C_{\max }^{*}$ and, hence,

$$
\frac{p_{j}}{s_{i_{0}}} \leq C_{\max }^{*}
$$

as $s_{i_{0}} \geq s_{i^{*}}$. Due to Lemma 10 , the load that would be contributed by job $j$ on machine $i_{2}$ is bounded by $p_{j} / s_{i_{2}}>(k-(k-4)-2) \cdot C_{\max }^{*}=2 \cdot C_{\max }^{*}$. Inequality (6) yields $s_{i_{0}} \geq 2 \cdot s_{i_{2}}$ as claimed in the lemma.

We want to show that machines in low classes, i.e., machines in $R_{0} \cup R_{1}$, have exponentially small speeds (with respect to $c$ ) compared to the speeds of the machines in a high class, i.e., those in $R_{c}$. Lemma 12 already implies that the machine speeds would double every five classes if no class $R_{k}$ was empty. Although some classes $R_{k}$ can be empty, we show that not too many of these machine classes are empty. This is done in Lemma 14 which follows from the next lemma.

The machines $i \in H_{k}, k \geq 2$, are overloaded compared to an optimal schedule, even if we just consider the first few jobs $j \in J_{i, \geq t}$ on them (where $t \geq 2$ ). On the other hand, in Corollary 11] we showed that in any optimal schedule these jobs are not assigned to machines in much lower classes, i.e., to machines from $M \backslash H_{k-t-1}$. Consequently, in any optimal schedule the machines in $H_{k-t-1} \backslash H_{k}$ consume the current overload of $H_{k}$.

Lemma 13. Let $t \leq k$ be positive integers. In any optimal schedule the total processing requirement on all machines in $H_{k-t-1} \backslash H_{k}$ is at least

$$
\sum_{k^{\prime}=k}^{c} \sum_{i \in R_{k^{\prime}}}\left(t+k^{\prime}-k-1\right) \cdot s_{i} \cdot C_{\max }^{*}
$$

Note that Lemma 13 also holds for the case $t=k$ where $H_{k-t-1}=H_{-1}=M$.

Proof. Applying Corollary 11 with $t^{\prime}\left(k^{\prime}\right)=t+\left(k^{\prime}-k\right)$ for arbitrary integers $k^{\prime} \in\{k, \ldots, c\}$ yields that in any optimal schedule $\sigma^{*}$ all jobs in $\bigcup_{k^{\prime}=k}^{c} \bigcup_{i \in R_{k^{\prime}}} J_{i, \geq t^{\prime}\left(k^{\prime}\right)}$ are assigned to machines in $H_{k-t-1}$ as $k^{\prime}-t^{\prime}\left(k^{\prime}\right)-1=k-t-1$ for any index $k^{\prime}$. Furthermore, in $\sigma^{*}$ the processing requirement on any machine $i \in H_{k}$ is at most $s_{i} \cdot C_{\text {max }}^{*}$, i.e., the machines in $H_{k-t-1} \backslash H_{k}$ must consume the remainder. Hence, these machines must process jobs with total processing requirement at least

$$
\sum_{k^{\prime}=k}^{c} \sum_{i \in R_{k^{\prime}}} \sum_{\ell \in J_{i, \geq t^{\prime}\left(k^{\prime}\right)}} p_{\ell}-\sum_{k^{\prime}=k}^{c} \sum_{i \in R_{k^{\prime}}} s_{i} \cdot C_{\max }^{*} \geq \sum_{k^{\prime}=k}^{c} \sum_{i \in R_{k^{\prime}}}\left(t^{\prime}\left(k^{\prime}\right)-1\right) \cdot s_{i} \cdot C_{\max }^{*} .
$$

This yields the claimed bound as $t^{\prime}\left(k^{\prime}\right)-1=t+k^{\prime}-k-1$. 
Although some machine classes $R_{k}$ might be empty, we are able to show that this cannot be the case for two consecutive classes.

Lemma 14. $H_{k-2} \backslash H_{k} \neq \emptyset$ for any $k \in\{1, \ldots, c-1\}$.

Proof. Let $i^{\prime}$ be a slowest machine in $H_{k}$. In any optimal schedule $\sigma^{*}$ the processing requirement on any machine $i \in H_{k-2} \backslash H_{k}$ is at most $s_{i} \cdot C_{\max }^{*} \leq s_{i^{\prime}} \cdot C_{\max }^{*}$. Applying Lemma 13 with $t=1$ implies

$$
\left|H_{k-2} \backslash H_{k}\right| \cdot s_{i^{\prime}} \cdot C_{\max }^{*} \geq \sum_{k^{\prime}=k}^{c} \sum_{i \in R_{k^{\prime}}}\left(k^{\prime}-k\right) \cdot s_{i} \cdot C_{\max }^{*} \geq \sum_{k^{\prime}=k}^{c}\left(k^{\prime}-k\right) \cdot s_{i^{\prime}} \cdot C_{\max }^{*} \cdot\left|R_{k^{\prime}}\right| .
$$

It follows that

$$
\left|H_{k-2} \backslash H_{k}\right| \geq \sum_{k^{\prime}=k}^{c}\left(k^{\prime}-k\right) \cdot\left|R_{k^{\prime}}\right| \geq(c-k) \cdot\left|R_{c}\right| \geq 1
$$

since $k<c$ and since $R_{c} \neq \emptyset$ due to Lemma 9 .

We can now show that machine speeds double every six classes. To be more formal:

Lemma 15. Let $0 \leq k_{2} \leq k_{1} \leq c$ be integers, let $i_{1}$ be any machine of $R_{k_{1}}$ and let $i_{2} \in R_{k_{2}}$. Then, $s_{i_{1}} \geq s_{i_{2}} \cdot 2^{\lfloor\Delta / 6\rfloor}$ where $\Delta=k_{1}-k_{2}$.

Proof. We prove the claim by induction. For $\Delta \in\{0, \ldots, 5\}$, the claim trivially holds as $s_{i_{1}} \geq s_{i_{2}}$. Assume that the claim holds up to some integer $\Delta^{*} \geq 5$. We show that it is also true for $\Delta=\Delta^{*}+1 \geq 6$. Note that for such $\Delta$ we have $k_{1} \geq 6$. According to Lemma 14 the class $H_{k_{1}-6} \backslash H_{k_{1}-4} \subseteq M \backslash H_{k_{1}-4}$ contains at least one machine. Let $i^{\prime}$ be the fastest machine in $H_{k_{1}-6} \backslash H_{k_{1}-4}$. Then $s_{i^{\prime}} \geq s_{i_{2}}$. Lemma 12 and the induction hypothesis imply $s_{i_{1}} \geq 2 s_{i^{\prime}}$ and $s_{i^{\prime}} \geq s_{i_{2}} \cdot 2^{\lfloor(\Delta-6) / 6\rfloor}$, respectively. Hence, $s_{i_{1}} \geq s_{i_{2}} \cdot 2^{\lfloor\Delta / 6\rfloor}$.

Since the machines in low classes are exponentially slower than the machines in high classes (with respect to $c$ ) and as their aggregated total processing requirement in an optimal schedule is large (Lemma 13), it follows that many jobs have processing requirements exponentially small in $c$.

Lemma 16. Let $i \in M \backslash H_{2}$ be an arbitrary machine. Then each job $j$ assigned to machine $i$ by an optimal schedule has processing requirement at most $p_{j} \leq 2^{-c / 6+2}$.

Proof. For $c \leq 12$ the claim is true since we rescale all processing requirements to be at most 1. Assume $c \geq 13$. Consider an optimal schedule $\sigma^{*}$ and let $j$ be a job processed on a machine $i \in M \backslash H_{2}=R_{1} \cup R_{0}$ according to $\sigma^{*}$. Note that $M \backslash H_{2} \neq \emptyset$ due to Lemma 14 . Then, $p_{j} / s_{i} \leq C_{\max }^{*}$, i.e.,

$$
p_{j} \leq s_{i} \cdot C_{\max }^{*} .
$$

To bound $s_{i} \cdot C_{\max }^{*}$, consider the job $j^{\prime}=\min \left\{\ell \in J_{1}(\sigma)\right\}$ with the smallest index on machine 1 of schedule $\sigma$ and consider the first machine $i^{\prime} \in H_{c-3} \backslash H_{c-1}=R_{c-3} \cup R_{c-2}$ which exists due to Lemma 14 and $c \geq 13$. Applying Inequality (5), we obtain $L_{i^{\prime}}(\sigma)+$ 
$p_{j^{\prime}} / s_{i^{\prime}} \geq L_{1}(\sigma)$, i.e., $p_{j^{\prime}} \geq s_{i^{\prime}} \cdot\left(L_{1}(\sigma)-L_{i^{\prime}}(\sigma)\right)$. Since machine 1 belongs to $H_{c}$ due to Lemma 9 and since machine $i^{\prime}$ is the first machine that does not belong to $H_{c-1}$, we have $L_{1}(\sigma) \geq c \cdot C_{\max }^{*}$ and $L_{i^{\prime}}(\sigma)<(c-1) \cdot C_{\max }^{*}$, which implies $p_{j^{\prime}} \geq s_{i^{\prime}} \cdot C_{\max }^{*}$. Lemma 15 yields $s_{i^{\prime}} \geq s_{i} \cdot 2^{\lfloor(c-3-1) / 6\rfloor}$. Applying Inequality 7 and $p_{j^{\prime}} \leq 1$ according to our input model we obtain

$$
p_{j} \leq s_{i} \cdot C_{\max }^{*} \leq s_{i^{\prime}} \cdot C_{\max }^{*} \cdot 2^{-\lfloor(c-4) / 6\rfloor} \leq p_{j^{\prime}} \cdot 2^{-c / 6+2} \leq 2^{-c / 6+2} .
$$

Corollary 17. The processing requirement of at least $n / 2$ jobs is at most $2^{-c / 6+2}$.

Proof. Lemma 13 for $k=t=2$ implies that the total processing requirement of all jobs assigned to machines from $M \backslash H_{2}=H_{-1} \backslash H_{2}$ according to $\sigma^{*}$ is at least $\sum_{i \in H_{2}} s_{i} \cdot C_{\max }^{*}$ which is an upper bound for the total processing requirement of all jobs assigned to machines in $H_{2}$ according to $\sigma^{*}$. Since all jobs assigned to machines from $M \backslash H_{2}$ by an optimal schedule have processing requirement at most $2^{-c / 6+2}$ due to Lemma 16 , at least half of the jobs have processing requirement at most $2^{-c / 6+2}$.

Since having many so small jobs is unlikely when the processing requirements have been smoothed, it follows that the smoothed performance guarantee, which is between $c+1$ and $c+2$, cannot be too high, yielding Theorem 6 .

Proof of Theorem 6. If $C_{\max }(\sigma) / C_{\max }^{*} \geq \alpha$, then at least $n / 2$ jobs have processing requirement at most $2^{-\alpha / 6+3}$ due to Corollary 17 and $c=\left\lfloor C_{\max }(\sigma) / C_{\max }^{*}\right\rfloor-1 \geq \alpha-2$. The probability that one specific job is that small is bounded by $\phi \cdot 2^{-\alpha / 6+3}=8 \phi \cdot 2^{-\alpha / 6}$ in the smoothed input model. Hence, the probability that the processing requirement of at least $n / 2$ jobs is at most $2^{-\alpha / 6+3}$, is bounded from above by

$$
\begin{aligned}
& \sum_{k \geq \frac{n}{2}}\left(\begin{array}{l}
n \\
k
\end{array}\right)\left(8 \phi \cdot 2^{-\alpha / 6}\right)^{k} \cdot\left(1-8 \phi \cdot 2^{-\alpha / 6}\right)^{n-k} \leq \sum_{k \geq \frac{n}{2}}\left(\begin{array}{l}
n \\
k
\end{array}\right)\left(8 \phi \cdot 2^{-\alpha / 6}\right)^{n / 2} \\
& \quad \leq 2^{n} \cdot\left(8 \phi \cdot 2^{-\alpha / 6}\right)^{n / 2}=\left(32 \phi \cdot 2^{-\alpha / 6}\right)^{n / 2} .
\end{aligned}
$$

Note that the first inequality holds if $8 \phi \cdot 2^{-\alpha / 6}<1$. Otherwise, the bound is trivially true. This yields

$$
\underset{I \sim \mathcal{I}}{\operatorname{Pr}}\left[\max _{\sigma \in \mathrm{NL}(I)} \frac{C_{\max }(I, \sigma)}{C_{\max }^{*}(I)} \geq \alpha\right] \leq\left(\frac{32 \phi}{2^{\alpha / 6}}\right)^{n / 2} .
$$

As for $n=1$ any schedule $\sigma \in \mathrm{NL}(I)$ is optimal, we just consider the case $n \geq 2$. For $k \geq 1$ let $\alpha_{k}=\alpha_{k}(\phi)=6 k \log _{2} \phi+30$, i.e., $2^{\alpha_{k} / 6}=32 \phi^{k}$. If $\alpha \geq \alpha_{k}$, then we obtain

$$
\begin{aligned}
\operatorname{Pr}_{I \sim \mathcal{I}}\left[\max _{\sigma \in \operatorname{NL}(I)} \frac{C_{\max }(I, \sigma)}{C_{\max }^{*}(I)} \geq \alpha\right] & \leq \underset{I \sim \mathcal{I}}{\operatorname{Pr}}\left[\max _{\sigma \in \operatorname{NL}(I)} \frac{C_{\max }(I, \sigma)}{C_{\max }^{*}(I)} \geq \alpha_{k}\right] \\
& \leq\left(\phi^{1-k}\right)^{n / 2} \leq \phi^{1-k} \leq 2^{1-k}
\end{aligned}
$$

as $\phi \geq 2$. Since $\alpha_{k+1}-\alpha_{k}=6 \log _{2} \phi$ we obtain

$$
\underset{I \sim \mathcal{I}}{\mathbf{E}}\left[\max _{\sigma \in \mathrm{NL}(I)} \frac{C_{\max }(I, \sigma)}{C_{\max }^{*}(I)}\right]=\int_{0}^{\infty} \underset{I \sim \mathcal{I}}{\operatorname{Pr}}\left[\max _{\sigma \in \mathrm{NL}(I)} \frac{C_{\max }(I, \sigma)}{C_{\max }^{*}(I)} \geq \alpha\right] \mathrm{d} \alpha
$$




$$
\begin{aligned}
& \leq \alpha_{1}+\sum_{k=1}^{\infty} \int_{\alpha_{k}}^{\alpha_{k+1}} \underset{I \sim \mathcal{I}}{\operatorname{Pr}}\left[\max _{\sigma \in \mathrm{NL}(I)} \frac{C_{\max }(I, \sigma)}{C_{\max }^{*}(I)} \geq \alpha\right] \mathrm{d} \alpha \\
& \leq \alpha_{1}+6 \log _{2} \phi \cdot \sum_{k=1}^{\infty} 2^{1-k}=18 \log _{2} \phi+30 .
\end{aligned}
$$

\subsection{Lower Bounds for List Schedules and Lex-jump Optimal Schedules}

In this subsection, we show that the upper bound, given in Theorem 6, on the smoothed performance guarantee on lex-jump optimal as well as list schedules is tight up to a constant factor. We provide a $\phi$-smooth instance such that the worst lex-jump optimal schedule as well as the worst schedule that can be obtained by list scheduling has a lower bound on the performance guarantee of $\Omega(\log \phi)$, for any realization of the processing times.

Theorem 18. There is a class of $\phi$-smooth instances $\mathcal{I}$ with unrestricted and related machines such that, for any $I \in \mathcal{I}$,

$$
\max _{\sigma \in \operatorname{Lex}(I)} \frac{C_{\max }(I, \sigma)}{C_{\max }^{*}(I)}=\Omega(\log \phi) \quad \text { and } \quad \max _{\sigma \in \operatorname{List}(I)} \frac{C_{\max }(I, \sigma)}{C_{\max }^{*}(I)}=\Omega(\log \phi) .
$$

To prove this theorem, we first present a $\phi$-smooth instance and in Algorithm 1, we implicitely give a permutation of the jobs such that list scheduling using this permutation results in a schedule $\sigma$ which we will show is also lex-jump optimal. The schedule $\sigma$ resembles the worst case example constructed by Czumaj and Vöcking [7]: Machines are partitioned into classes indexed by $0,1, \ldots, r$. We will show that in $\sigma$, each machine in class $i$ has a load of approximately $i$, whereas the optimal makespan is bounded by 3 . Hence, we can lower bound the performance guarantee in the order of the number of classes. Whereas Czumaj and Vöcking needed $\Theta(\log m / \log \log m)$ classes, we only need $\Theta(\log \phi)$ classes.

As scaling of all processing requirements does not change the approximation ratio, for sake of simplicity we do not consider probability densities $f_{j}:[0,1] \rightarrow[0, \phi]$ but scaled densities $f_{j}^{\prime}:\left[0,2^{r+1}\right] \rightarrow\left[0, \phi / 2^{r+1}\right]$ for an appropriate integer $r$.

Let $\phi \geq 4$ and consider an integer $r=\left\lfloor\log _{4} \phi\right\rfloor \geq 1$, i.e., $\phi \geq 4^{r}=2^{2 r}$. The machines are partitioned into machine classes $M_{k}$ for $k=0, \ldots, r$, such that machine class $M_{k}$ contains $r ! / k$ ! machines of speed $2^{k}$. Also the jobs are partitioned into job classes $J_{\ell}$ for $\ell=1, \ldots, r$ such that a job class $J_{\ell}$ contains $r ! /(\ell-1)$ ! jobs each having a processing requirement uniformly drawn from $\left[2^{\ell}, 2^{\ell}+2^{r+1} / \phi\right) \subseteq\left(0,2^{r+1}\right)$. Note that the density of this instance is bounded by $\phi / 2^{r+1}$ which is valid in the variant of our model that we use in this subsection. The permutation of the jobs is such that list scheduling constructs the schedule $\sigma$ in the following way:

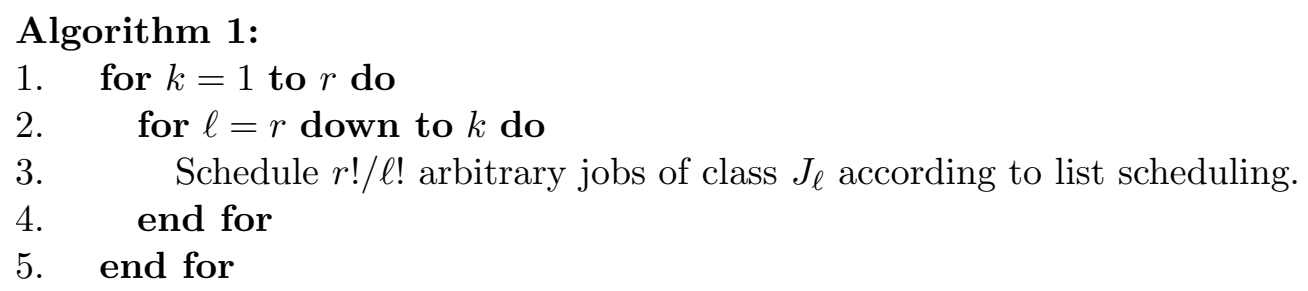


Note that for any job class $J_{\ell}$ all $\ell \cdot r ! / \ell !=r ! /(\ell-1) !$ jobs have been scheduled. Let $\sigma$ be the resulting schedule. First, we show a key property of $\sigma$.

Lemma 19. For any index $\ell=1, \ldots, r$ each machine in $M_{\ell}$ is assigned exactly $\ell$ jobs of job class $J_{\ell}$ and no other jobs. The machines in $M_{0}$ remain empty.

Proof. Let $\sigma(k, \ell)$ denote the partial schedule after processing line 3 of iteration $(k, \ell)$ of Algorithm 1. Within the $(k, \ell)^{t h}$ iteration, we call a machine $i \in M_{\ell}$ used if a job of class $J_{\ell}$ has already been assigned to $i$ during that iteration. Otherwise, we call machine $i$ unused. We show the two claims below inductively and simultaneously. The lemma then follows straightforwardly from the second claim since the last iteration is $(r, r)$.

Claim 1. During iteration $(k, \ell), r ! / \ell$ ! jobs of class $J_{\ell}$ are assigned to $r ! / \ell$ ! distinct machines (i.e. all machines) of class $M_{\ell}$.

Claim 2. In the partial schedule $\sigma(k, \ell)$ each machine in class $M_{\ell^{\prime}}$ is assigned

$$
k^{\prime}=\left\{\begin{array}{ccc}
k & : \quad \ell^{\prime} \geq \ell, \\
\min \left\{k-1, \ell^{\prime}\right\} & : \quad \ell^{\prime}<\ell,
\end{array}\right.
$$

jobs of class $J_{\ell^{\prime}}$ and no other jobs.

Figure 6 visualizes the partial schedule $\sigma(k, \ell)$. Machine $i$ with speed $s_{i}=2^{i}$ is a representative for all machines in class $M_{i}$. With $L_{i}$ we refer to the current load of machine $i$ and with $L_{i}^{\prime}$ to the load of machine $i$ at the end of iteration $(k, k)$, i.e., in the partial schedule $\sigma(k, k)$. In phase $(k, \ell), r ! / \ell$ ! jobs of size roughly $2^{\ell}$ are being assigned to the $r ! / \ell$ ! machines in $M_{\ell}$. All machines in $M_{\ell^{\prime}}$ for $\ell^{\prime}>\ell$ just received a job of roughly size $2^{\ell^{\prime}}$. All machines in $M_{\ell^{\prime}}$ for $\ell^{\prime} \in\{k, \ldots, \ell-1\}$ will still receive a single job of size roughly $2^{\ell^{\prime}}$ during iteration $k$ of the outer loop. Figure 6 follows from the observations.

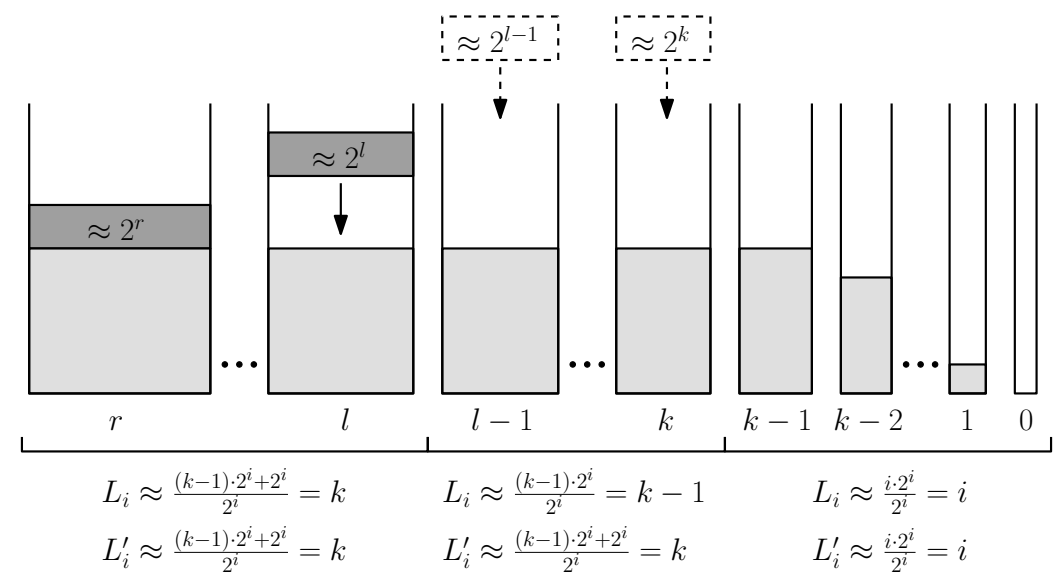

Figure 6: The partial schedule $\sigma(k, \ell)$

First, we validate the claims for the first iteration $(1, r)$. As only $r ! / r !=1$ job of class $J_{r}$ has to be scheduled and since all machines are still empty, the job will be scheduled on the fastest machine which is the single machine in $M_{r}$. Hence, both claims hold true for 
the first iteration. Now, consider an arbitrary iteration $(k, \ell)$ and assume both claims hold true for all previous iterations. Consider a job $j \in J_{\ell}$ which needs to be assigned to a machine during iteration $(k, \ell)$. We show that job $j$ will always be assigned to an unused machine $i \in M_{\ell}$. To see this, first note that the previous iteration was either $(k, \ell+1)$ or $(k-1, k-1)$.

Let $i \in M_{\ell}$ be an unused machine. By the second claim, we know that this machine carries $k-1$ jobs of class $J_{\ell}$. Consequently, we can upper bound its load by

$$
L_{i}+\frac{p_{j}}{s_{i}}<\frac{k \cdot\left(2^{\ell}+2^{r+1} / \phi\right)}{2^{\ell}}=k+\frac{k}{\phi} \cdot 2^{r+1-\ell} \leq k+\frac{\ell}{2^{2 r}} \cdot 2^{r+1-\ell} \leq k+\frac{1}{2^{r}},
$$

where we used that $k \leq \ell, \phi \geq 2^{2 r}$, and $\ell / 2^{\ell} \leq 1 / 2$ for all integers $\ell \geq 1$.

Consider a machine machine $h$ which is either used (in that case let $\ell^{\prime}=\ell$ ) or in class $M_{\ell^{\prime}}$ for some $\ell^{\prime} \in\{\ell+1, \ldots, r\}$. By Claim 2 , this machine carries $k$ jobs of class $J_{\ell^{\prime}}$ and thus

$$
L_{h}+\frac{p_{j}}{s_{h}} \geq \frac{k \cdot 2^{\ell^{\prime}}+2^{\ell}}{2^{\ell^{\prime}}}=k+2^{\ell-\ell^{\prime}}>k+\frac{1}{2^{r}}>L_{i}+\frac{p_{j}}{s_{i}} .
$$

Finally, consider a machine $h \in M_{\ell^{\prime}}$ for some $\ell^{\prime} \in\{1, \ldots, \ell-1\}$. Again by Claim 2 , it carries $\min \left\{k-1, \ell^{\prime}\right\}$ jobs of class $J_{\ell^{\prime}}$ and thus

$$
\begin{aligned}
L_{h}+\frac{p_{j}}{s_{h}} & \geq \frac{\min \left\{k-1, \ell^{\prime}\right\} \cdot 2^{\ell^{\prime}}+2^{\ell}}{2^{\ell^{\prime}}}=\min \left\{k-1, \ell^{\prime}\right\}+2^{\ell-\ell^{\prime}} \\
& \geq\left(k-\max \left\{k-\ell^{\prime}, 1\right\}\right)+2^{\max \left\{k-\ell^{\prime}, 1\right\}} \geq k+1>L_{i}+\frac{p_{j}}{s_{i}},
\end{aligned}
$$

where the second inequality follows from $\ell \geq \max \left\{k, \ell^{\prime}+1\right\}$ and the third inequality follows from $2^{i}-i \geq 1$ for all positive integers $i$.

With this complete case analysis we have shown that job $j$ will be assigned to an unused machine $i \in M_{\ell}$. We conclude that during iteration $(k, \ell)$, each of the $r ! / \ell$ ! jobs to be assigned will be assigned to an unused machine in $M_{\ell}$. Note that $\left|M_{\ell}\right|=r ! / \ell$ !, and hence for each job there always exists such an unused machine. The first claim and the second claim follow immediately.

Lemma 20. Schedule $\sigma$ is lex-jump optimal.

Proof. It follows from Lemma 19 that the load of any machine $i \in M_{\ell}$ can be bounded by

$$
\ell \leq L_{i} \leq \ell+\ell \cdot \frac{2^{r+1}}{2^{\ell} \phi} \leq \ell+\frac{\ell}{2^{\ell}} \cdot \frac{2^{r+1}}{2^{2 r}} \leq \ell+\frac{1}{2} \cdot \frac{2^{r+1}}{2^{2 r}}<\ell+1 .
$$

If a job $j$ assigned to machine $i \in M_{\ell}$ would jump to another machine $i^{\prime} \in M_{\ell^{\prime}}$, then

$$
L_{i^{\prime}}+\frac{p_{j}}{s_{i^{\prime}}} \geq \ell^{\prime}+\frac{2^{\ell}}{2^{\ell^{\prime}}}=\ell-\left(\ell-\ell^{\prime}\right)+2^{\ell-\ell^{\prime}} \geq \ell+1>L_{i},
$$

where the last inequality follows from $2^{k}-k \geq 1$ for all integers $k$. Thus, any job would be worse off by jumping to another machine, and hence schedule $\sigma$ is lex-jump optimal. 
We conclude this subsection by proving Theorem 18 .

Proof of Theorem 18. We consider schedule $\sigma$ constructed above which is both a list schedule and a lex-jump optimal schedule. By Lemma 19 the load of the single machine in $M_{r}$ is at least $r$. Hence, $C_{\max }(\sigma) \geq r$. Now, consider a schedule $\sigma^{\prime}$ in which each machine in $M_{\ell}$ processes a single job from job class $J_{\ell+1}, \ell=0, \ldots, r-1$. The single machine in $M_{r}$ remains empty. Then, the load of any machine $i \in M_{\ell}$ with job $j$ assigned to it is bounded as follows:

$$
L_{i}=p_{j} / s_{i} \leq\left(2^{\ell+1}+2^{r+1} / \phi\right) / 2^{\ell} \leq 2+2^{r+1} /\left(2^{2 r} \cdot 2^{1}\right)=2+2^{-r}<3 .
$$

Hence, $C_{\max }^{*}(I) \leq C_{\max }\left(I, \sigma^{\prime}\right)<3$ and the theorem follows: $C_{\max }(\sigma) / C_{\max }^{*}(I) \geq r / 3=$ $\Omega(r)=\Omega(\log \phi)$.

\section{Restricted Machines}

In this section, we provide lower bound examples showing that the worst-case performance guarantees for all variants of the restricted machines are robust against random noise. Our lower bounds are in the order of the worst-case bounds and hold in particular for $\phi=2$. In our lower bound constructions all processing requirements are chosen uniformly at random from intervals of length $1 / 2$. This means that even with large perturbations the worst-case lower bounds still apply.

\subsection{Jump Neighborhood on Restricted Machines}

Rutten et al. [21] showed that the makespan of a jump optimal schedule is at most a factor of $1 / 2+\sqrt{m-3 / 4}$ away from the optimal makespan on restricted identical machines. On restricted related machines they showed that the makespan of a jump optimal schedule is not more than a factor of $1 / 2+\sqrt{(m-1) \cdot s_{\max }+1 / 4}$ away from the makespan of an optimal schedule, assuming that $s_{\min }=1$. They provided two examples showing that the bound on identical machines is tight and the one on related machines is tight up to a constant factor. We show that even on $\phi$-smooth instances these bounds are tight up to a constant factor. As in [21], we construct an example with two job classes and three machine classes. The first machine class consists of only one machine and this machine is the slowest among all machines. The first class of jobs can only be scheduled on machines in the first two classes, whereas the jobs in the second class are allowed on all machines. To construct a bad example, we schedule all jobs in the first class on the slowest machine and use the jobs of the second class to fill the machines in the second machine class so that the schedule will be jump optimal, with high probability.

Theorem 21. For every $\phi \geq 2$ there exists a class of $\phi$-smooth instances $\mathcal{I}$ on restricted related machines such that

$$
\underset{I \sim \mathcal{I}}{\mathbf{E}}\left[\max _{\sigma \in \operatorname{Jump}(I)} \frac{C_{\max }(I, \sigma)}{C_{\max }^{*}(I)}\right]=\Omega\left(\sqrt{m \cdot s_{\max }}\right),
$$

assuming without loss of generality that $s_{\min }=1$. 
Proof. It suffices to show the theorem for $\phi=2$ and $m \geq 3$. W.l.o.g. we assume $s_{\min }=1$ and set $s:=s_{\max } / s_{\min }=s_{\max }$. Let $z>2$ be an arbitrary integer, let

$$
m^{\prime}=m-2 \geq 1, \quad k^{\prime}=\sqrt{\frac{m^{\prime}}{s}} \leq \sqrt{m^{\prime}}, \quad \text { and } \quad k=\left\lceil k^{\prime}\right\rceil .
$$

In the remainder we assume that $\sqrt{m^{\prime} s} \geq 17$. This is possible because we only want to derive an asymptotic bound. We consider the following $\phi$-smooth instance $\mathcal{I}$. The set $M$ of machines is partitioned into three classes $M_{1}, M_{2}$, and $M_{3}$ such that

$$
\left|M_{1}\right|=1, \quad\left|M_{2}\right|=k, \quad \text { and } \quad\left|M_{3}\right|=m^{\prime}-(k-1)>m^{\prime}-k^{\prime} \geq 0 .
$$

The machine in $M_{1}$ has speed 1, the machines in $M_{2}$ have speed

$$
s^{\prime}=\max \left\{1, s \cdot \frac{k^{\prime}}{k}\right\} \in[1, s],
$$

and the machines in $M_{3}$ have speed $s$. Let the set $J$ of jobs be partitioned into two subsets $\mathcal{J}_{1}$ and $\mathcal{J}_{2}$, consisting of

$$
\left|\mathcal{J}_{1}\right|=\left\lfloor 2 z s k^{\prime}\right\rfloor \quad \text { and } \quad\left|\mathcal{J}_{2}\right|=\left\lceil 32 z s \cdot\left(m^{\prime}-k^{\prime}\right)\right\rceil \leq\left\lceil 32 z s \cdot\left|M_{3}\right|\right\rceil
$$

jobs whose processing requirements are independently and uniformly drawn from $[1 / 2,1]$ and from $[0,1 / 2]$, respectively. The jobs in $\mathcal{J}_{1}$ are only allowed to be scheduled on the machines in $M_{1} \cup M_{2}$, whereas the jobs in $\mathcal{J}_{2}$ are allowed to be scheduled on any machine.

First, we construct a schedule $\sigma^{\prime}$ to bound the optimal makespan: Use the list scheduling algorithm to schedule all jobs in $\mathcal{J}_{1}$ on the machines in $M_{2}$, and all jobs in $\mathcal{J}_{2}$ on the machines in $M_{3}$. Figure 7 depicts schedule $\sigma^{\prime}$. Machine $i$ is a representative for all machines in class $M_{i}$.

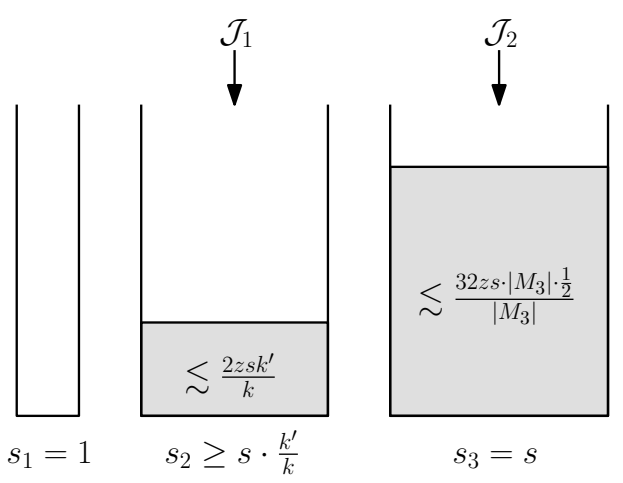

Figure 7: Schedule $\sigma^{\prime}$

Along the same lines as in [12], it follows that for all machines $i \in M_{2}$

$$
L_{i} \leq \frac{\frac{\sum_{j \in \mathcal{J}_{1}} p_{j}}{\left|M_{2}\right|}+\max _{j \in \mathcal{J}_{1}} p_{j}}{s^{\prime}} \leq \frac{\frac{\left|\mathcal{J}_{1}\right| \cdot 1}{\left|M_{2}\right|}+1}{s^{\prime}} \leq \frac{\frac{2 z s k^{\prime}}{k}+1}{s^{\prime}} \leq \frac{\frac{2 z s k^{\prime}}{k}}{s \cdot \frac{k^{\prime}}{k}}+\frac{1}{1}=2 z+1 .
$$


Similarly, for all machines $i \in M_{3}$

$$
L_{i} \leq \frac{\frac{\sum_{j \in \mathcal{J}_{2}} p_{j}}{\left|M_{3}\right|}+\max _{j \in \mathcal{J}_{2}} p_{j}}{s} \leq \frac{\frac{\left|\mathcal{J}_{2}\right| \cdot \frac{1}{2}}{\left|M_{3}\right|}+\frac{1}{2}}{s} \leq \frac{\frac{32 z s \cdot\left|M_{3}\right|}{2 \cdot\left|M_{3}\right|}+1}{s} \leq 16 z+1 .
$$

Hence, $C_{\max }^{*} \leq C_{\max }\left(\sigma^{\prime}\right) \leq 17 z$. Before we proceed with constructing a 'bad' jump optimal schedule $\sigma$, we observe that

$$
s^{\prime} \leq 2 s \cdot k^{\prime} / k
$$

due to $1 \leq\left(\sqrt{m^{\prime}}+1\right) / k \leq 2 \sqrt{m^{\prime} s} / k=2 s \cdot k^{\prime} / k$.

We construct a jump optimal schedule $\sigma$ on the $\phi$-smooth instance $\mathcal{I}$ such that the corresponding makespan exceeds $z s k^{\prime}$ with high probability: Schedule all jobs in $\mathcal{J}_{1}$ on the single machine in $M_{1}$. Then, $z s k^{\prime}-1 \leq L_{1} \leq 2 z s k^{\prime}$. Next, start assigning jobs from $\mathcal{J}_{2}$ to the machines in $M_{2}$ according to the list scheduling algorithm with an arbitrary job permutation, until

(a) either $\mathcal{J}_{2}$ becomes empty, or until

(b) $L_{i} \in\left[L_{1}-\frac{1}{2 s^{\prime}}, L_{1}\right)$ for all $i \in M_{2}$. If there remain unscheduled jobs in $\mathcal{J}_{2}$, then we assign them to the machines in $M_{3}$ using list scheduling.

Let $Q=\sum_{j \in \mathcal{J}_{2}} p_{j}$ and let $\mathcal{E}$ denote the event that $Q>4 z\left(s k^{\prime}\right)^{2}$. If $\mathcal{E}$ occurs, then

$$
\sum_{i \in M_{2}} s^{\prime} \cdot L_{1} \leq\left|M_{2}\right| \cdot\left(2 s \cdot \frac{k^{\prime}}{k}\right) \cdot 2 z s k^{\prime}=4 z\left(s k^{\prime}\right)^{2}<Q
$$

due to Inequality (8), i.e., the algorithm will end up in case (b) as $p_{j} \leq 1 / 2$ for any job $j \in \mathcal{J}_{2}$. This shows that no machine $i \in M_{2}$ is critical. Using the same argument as for the analysis of $\sigma^{\prime}$ we can show that the load of any machine $i \in M_{3}$ is bounded from above by $16 z+1<17 z-1 \leq z \cdot \sqrt{m^{\prime} \cdot s}-1=z s k^{\prime}-1 \leq L_{1}$, i.e., the machine in $M_{1}$ is the unique critical machine. As each job on this machine has processing requirement at least $1 / 2$ and due to the property of the loads of the machines in $M_{2}$ in case (b), schedule $\sigma$ is jump optimal and $C_{\max }(\sigma)=L_{1} \geq z s k^{\prime}-1$.

It remains to determine the probability $\operatorname{Pr}[\mathcal{E}]$. For this, note that

$$
\begin{aligned}
\mathbf{E}[Q] & =\frac{\left|\mathcal{J}_{2}\right|}{4} \geq 8 z s \cdot\left(m^{\prime}-k^{\prime}\right)=8 z s m^{\prime} \cdot\left(1-\frac{k^{\prime}}{m^{\prime}}\right) \\
& =8 z s m^{\prime} \cdot\left(1-\frac{1}{\sqrt{m^{\prime} s}}\right)>6 z s m^{\prime}
\end{aligned}
$$

as $\sqrt{m^{\prime} s} \geq 17$ by our initial assumption. On the other hand, $4 z\left(s k^{\prime}\right)^{2}=4 z s m^{\prime}$. Applying Hoeffding's Inequality [16] (see also Theorem 31, we obtain

$$
\begin{aligned}
\operatorname{Pr}[\overline{\mathcal{E}}] & =\operatorname{Pr}\left[Q \leq 4 z s m^{\prime}\right] \leq \operatorname{Pr}\left[Q-\mathbf{E}[Q] \leq-2 z s m^{\prime}\right] \\
& \leq \exp \left(-\frac{2 \cdot\left(2 z s m^{\prime}\right)^{2}}{\left|\mathcal{J}_{2}\right| \cdot\left(\frac{1}{2}\right)^{2}}\right) \leq \exp \left(-\frac{32 z^{2} s^{2} m^{\prime 2}}{32 z s m^{\prime}+1}\right),
\end{aligned}
$$


which becomes arbitrarily close to 0 when $z$ increases. Hence, for sufficiently large integers $z$

$$
\begin{aligned}
\underset{I \sim \mathcal{I}}{\mathbf{E}}\left[\max _{\sigma \in \operatorname{Jump}(I)} \frac{C_{\max }(I, \sigma)}{C_{\max }^{*}(I)}\right] & \geq \underset{I \sim \mathcal{I}}{\mathbf{E}}\left[\max _{\sigma \in \operatorname{Jump}(I)} \frac{C_{\max }(I, \sigma)}{C_{\max }^{*}(I)} \mid \mathcal{E}\right] \cdot \underset{I \sim \mathcal{I}}{\operatorname{Pr}}[\mathcal{E}] \\
& \geq \frac{z s k^{\prime}-1}{17 z} \cdot \frac{17}{18} \geq \frac{\sqrt{(m-2) \cdot s_{\max }}-\frac{1}{z}}{18} .
\end{aligned}
$$

Corollary 22. For every $\phi \geq 2$ there exists a class of $\phi$-smooth instances $\mathcal{I}$ on restricted identical machines such that

$$
\underset{I \sim \mathcal{I}}{\mathbf{E}}\left[\max _{\sigma \in \mathrm{Jump}(I)} \frac{C_{\max }(I, \sigma)}{C_{\max }^{*}(I)}\right]=\Omega(\sqrt{m}) .
$$

Remark. In the proof of Theorem 21 we introduce an arbitrary integer $z$. We argue that there exists a sufficiently large value for $z$ such that the desired result follows. Choosing an even larger value for $z$ implies that the results above not only hold in expectation but also with high probability.

\subsection{Lex-jump Optimal Schedules on Restricted Identical Machines}

In this subsection, we show that there exist instances with $\phi \geq 8$ such that the smoothed performance guarantee for lex-jump optimal schedules in the restricted setting is in the same order as the worst case performance guarantee.

As in Section 2.3, we construct an instance with several job classes and machine classes and the loads of the machines are gradually decreasing with increasing machine class. By setting the sets $\mathcal{M}_{j}$ of allowed machines equal to the union of only one or two machine classes and choosing to schedule the jobs on the wrong machines, we can enforce that jobs cannot leave the machine class on which they are scheduled in the lex-jump optimal solution, whereas the optimal makespan is still small.

Theorem 23. For every $\phi \geq 8$ there exists a class of $\phi$-smooth instances $\mathcal{I}$ on restricted identical machines such that

$$
\underset{I \sim \mathcal{I}}{\mathbf{E}}\left[\max _{\sigma \in \operatorname{Lex}(I)} \frac{C_{\max }(I, \sigma)}{C_{\max }^{*}(I)}\right]=\Omega\left(\frac{\log m}{\log \log m}\right) .
$$

First, we introduce the $\phi$-smooth instance $\mathcal{I}$ for $\phi \geq 8$. Given an integer $k \geq 68$, consider the following recurrence formula:

$$
a_{0}=k^{2}, \quad a_{1}=k^{3}, \quad \text { and } \quad a_{h}=\left\lceil\left(\frac{a_{h-1}}{a_{h-2}}-\frac{7}{15}\right) \cdot a_{h-1}\right\rceil \text { for } h \geq 2 .
$$

Starting with $a_{1} / a_{0}=k$, the fraction $a_{h} / a_{h-1}$ decreases with increasing index $h$ until it is less or equal 1. To see this, note that $a_{h} / a_{h-1} \geq 1$ implies that $a_{h-1} \geq a_{h-2}$. Therefore, we know that $a_{h} \geq a_{h-1} \geq \ldots \geq a_{0}=k^{2}>15$. Furthermore, we can bound the ratio $a_{h} / a_{h-1}$ from above by $a_{h} / a_{h-1} \leq a_{h-1} / a_{h-2}-7 / 15+1 / a_{h-1}<a_{h-1} / a_{h-2}-6 / 15<a_{h-1} / a_{h-2}$. Let $z_{k}$ be the smallest integer $h$ such that $a_{h} / a_{h-1} \leq 1$. Hence, $a_{0}, a_{1}, \ldots, a_{z_{k}-1}$ is a strictly increasing sequence. We will bound the number $z_{k}$ from above later in the analysis. 
We consider $z_{k}$ job classes $\mathcal{J}_{1}, \ldots, \mathcal{J}_{z_{k}}$ and as many machine classes $M_{1}, \ldots, M_{z_{k}}$. Each machine class $M_{h}$ contains $m_{h}=a_{h-1}$ machines with speed 1 . Each job class $\mathcal{J}_{h}$ consists of two subclasses $\mathcal{J}_{h}^{A}$ and $\mathcal{J}_{h}^{B}$ of size $a_{h}$ and of size $b_{h}=17 m_{h}$, respectively. The jobs in class $\mathcal{J}_{h}^{A}$ are called type $A$ jobs, have processing requirements independently and uniformly distributed in [7/8,1], and can be processed on machines in $M_{h} \cup M_{h+1}$. As a convention let $M_{z_{k}+1}=\emptyset$. Jobs in class $\mathcal{J}_{h}^{B}$ are called type $B$ jobs, have processing requirements independently and uniformly distributed in $[0,1 / 8]$, and can only be processed on machines in $M_{h}$.

The schedule $\sigma=\sigma(I)$ for an instance $I \in \mathcal{I}$ is obtained by scheduling the jobs in $\mathcal{J}_{h}$ on the machines in $M_{h}$ using LPT (longest processing time) scheduling, i.e., list scheduling with a list in which the jobs are ordered according to non-increasing processing requirements. Note that the LPT algorithm first schedules all type A jobs and then all type B jobs. Schedule $\sigma(I)$ is visualized in Figure 8. Machine $h$ represents all machines in class $M_{h}$.

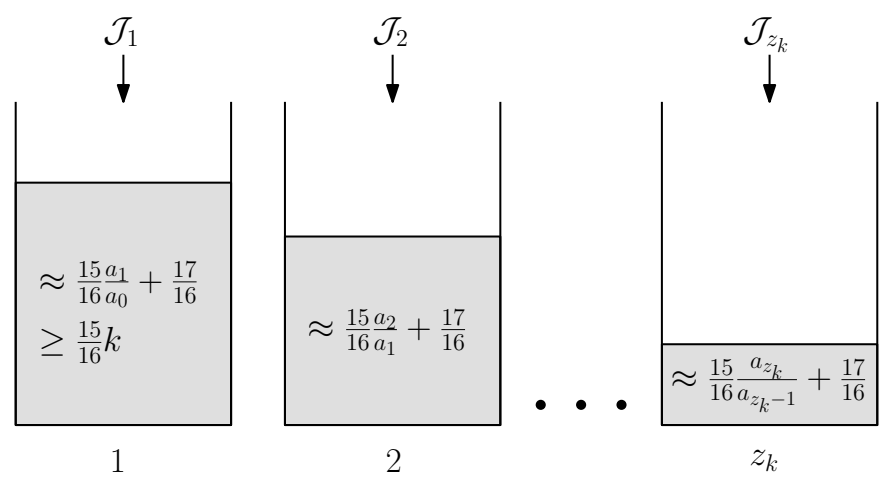

Figure 8: Schedule $\sigma(I)$

We show that schedule $\sigma$ is lex-jump optimal with high probability. To be more specific, we show lex-jump optimality when the values $Q_{h}^{A}=\sum_{j \in \mathcal{J}_{h}^{A}} p_{j}$ and $Q_{h}^{B}=\sum_{j \in \mathcal{J}_{h}^{B}} p_{j}$ are close to their expectations for all $h=1, \ldots, z_{k}$. Let $\mathcal{E}_{h}^{A}$ and $\mathcal{E}_{h}^{B}$ denote the events that

$$
\left|Q_{h}^{A}-\mathbf{E}\left[Q_{h}^{A}\right]\right| \leq \frac{m_{h}}{16} \quad \text { and } \quad\left|Q_{h}^{B}-\mathbf{E}\left[Q_{h}^{B}\right]\right| \leq \frac{m_{h}}{32}, \quad \text { respectively } .
$$

Moreover, let $\mathcal{E}$ denote the event that the events $\mathcal{E}_{h}^{A}$ and $\mathcal{E}_{h}^{B}$ are simultaneously true for all $h=1, \ldots, z_{k}$. By $\overline{\mathcal{E}}_{h}^{A}, \overline{\mathcal{E}}_{h}^{B}$, and $\overline{\mathcal{E}}$ we refer to the complement of $\mathcal{E}_{h}^{A}, \mathcal{E}_{h}^{B}$, and $\mathcal{E}$.

First, we analyze the sequence $a_{0}, a_{1}, \ldots, a_{z_{k}}$ to obtain bounds for the number $z_{k}$ of machine and job classes and for the number $m$ of machines.

Lemma 24. For any $h=1, \ldots, z_{k}$ the following inequality holds:

$$
\frac{a_{h}}{a_{h-1}} \leq k-(h-1) \cdot \frac{2}{5} \text {. }
$$

Proof. The claim is true for $h=1$. By definition of $a_{h}$,

$$
\frac{a_{h}}{a_{h-1}} \leq \frac{\left(\frac{a_{h-1}}{a_{h-2}}-\frac{7}{15}\right) \cdot a_{h-1}+1}{a_{h-1}} \leq \frac{a_{h-1}}{a_{h-2}}-\frac{6}{15}=\frac{a_{h-1}}{a_{h-2}}-\frac{2}{5}
$$


for any $h=2, \ldots, z_{k}$ as $a_{h-1} \geq a_{0}=k^{2} \geq 15$. The claim follows by induction.

Now, we can bound the number $z_{k}$ of job classes.

Corollary 25. The number $z_{k}$ of machine classes and job classes is bounded by $5 k / 2$.

Proof. Applying Lemma 24 for $h=z_{k}-1$ we obtain

$$
1<\frac{a_{z_{k}-1}}{a_{z_{k}-2}} \leq k-\left(z_{k}-2\right) \cdot \frac{2}{5} .
$$

Hence,

$$
z_{k}<(k-1) \cdot \frac{5}{2}+2<\frac{5 k}{2} .
$$

Lemma 26. The number $m$ of machines is bounded by $\Gamma\left(k^{\prime}+3\right)$ where $\Gamma$ denotes the gamma function and where $k^{\prime}=\lceil 5 k / 2\rceil$.

Proof. By induction we show that

$$
a_{h} \leq k^{2} \cdot\left(\frac{2}{5}\right)^{h} \cdot \frac{k^{\prime} !}{\left(k^{\prime}-h\right) !}
$$

for any $h=0, \ldots, z_{k}-1$. Note that $z_{k} \leq 5 k / 2 \leq k^{\prime}$ due to Corollary 25. For $h=0$ the claim holds since $a_{0}=k^{2}$. For $h \geq 1$ we apply Lemma 24 to get

$$
\frac{a_{h}}{a_{h-1}} \leq k-(h-1) \cdot \frac{2}{5} \leq \frac{2}{5} \cdot\left(k^{\prime}-(h-1)\right) .
$$

The induction hypothesis for $a_{h-1}$ yields

$$
a_{h} \leq \frac{2}{5} \cdot\left(k^{\prime}-(h-1)\right) \cdot k^{2} \cdot\left(\frac{2}{5}\right)^{h-1} \cdot \frac{k^{\prime} !}{\left(k^{\prime}-(h-1)\right) !}=k^{2} \cdot\left(\frac{2}{5}\right)^{h} \cdot \frac{k^{\prime} !}{\left(k^{\prime}-h\right) !} .
$$

Recalling $m_{h}=a_{h-1}$ we can bound the number $m$ of machines by using

$$
\frac{m}{k^{2}}=\sum_{h=1}^{z_{k}} \frac{m_{h}}{k^{2}}=\sum_{h=0}^{z_{k}-1} \frac{a_{h}}{k^{2}} \leq \sum_{h=0}^{z_{k}-1} \frac{k^{\prime} !}{\left(k^{\prime}-h\right) !} \leq k^{\prime} ! \cdot e .
$$

Hence, $m \leq e \cdot k^{2} \cdot k^{\prime} ! \leq\left(k^{\prime}+2\right) !=\Gamma\left(k^{\prime}+3\right)$.

Lemma 27. Event $\overline{\mathcal{E}}$ occurs with probability at most $10 k \cdot \exp (-k / 2)$.

Proof. We bound the probability for the events $\overline{\mathcal{E}}_{h}^{A}$ and $\overline{\mathcal{E}}_{h}^{B}$ to occur. Recalling $m_{h}=$ $a_{h-1} \geq a_{0}=k^{2}, a_{h} \leq k \cdot a_{h-1}$ (see Lemma 24 ), $b_{h}=17 m_{h}$, and $k \geq 68$ we obtain

$$
\begin{aligned}
\operatorname{Pr}\left[\overline{\mathcal{E}}_{h}^{A}\right] & =\operatorname{Pr}\left[\left|Q_{h}^{A}-\mathbf{E}\left[Q_{h}^{A}\right]\right|>\frac{m_{h}}{16}\right] \leq 2 \exp \left(-\frac{2\left(\frac{m_{h}}{16}\right)^{2}}{a_{h} \cdot\left(\frac{1}{8}\right)^{2}}\right) \\
& =2 \exp \left(-\frac{a_{h-1}}{a_{h}} \cdot \frac{a_{h-1}}{2}\right) \leq 2 \exp \left(-\frac{a_{h-1}}{2 k}\right) \leq 2 \exp \left(-\frac{k}{2}\right)
\end{aligned}
$$


and

$$
\begin{aligned}
\operatorname{Pr}\left[\overline{\mathcal{E}}_{h}^{B}\right] & =\operatorname{Pr}\left[\left|Q_{h}^{B}-\mathbf{E}\left[Q_{h}^{B}\right]\right|>\frac{m_{h}}{32}\right] \leq 2 \exp \left(-\frac{2\left(\frac{m_{h}}{32}\right)^{2}}{b_{h} \cdot\left(\frac{1}{8}\right)^{2}}\right) \\
& =2 \exp \left(-\frac{m_{h}}{17 m_{h}} \cdot \frac{a_{h-1}}{8}\right) \leq 2 \exp \left(-\frac{k^{2}}{136}\right) \leq 2 \exp \left(-\frac{k}{2}\right) .
\end{aligned}
$$

Each of the first inequalities stems from Hoeffding's bound [16] (see also Theorem 31). A union bound yields

$$
\operatorname{Pr}[\overline{\mathcal{E}}]=\operatorname{Pr}\left[\bigcup_{h=1}^{z_{k}}\left(\overline{\mathcal{E}}_{h}^{A} \cup \overline{\mathcal{E}}_{h}^{B}\right)\right] \leq 2 z_{k} \cdot 2 \exp \left(-\frac{k}{2}\right) \leq 10 k \cdot \exp \left(-\frac{k}{2}\right)
$$

due to Corollary 25.

As event $\mathcal{E}$ occurs with high probability and as

$$
\underset{I \sim \mathcal{I}}{\mathbf{E}}\left[\max _{\sigma \in \operatorname{Lex}(I)} \frac{C_{\max }(I, \sigma)}{C_{\max }^{*}(I)}\right] \geq \underset{I \sim \mathcal{I}}{\mathbf{E}}\left[\max _{\sigma \in \operatorname{Lex}(I)} \frac{C_{\max }(I, \sigma)}{C_{\max }^{*}(I)} \mid \mathcal{E}\right] \cdot \underset{I \sim \mathcal{I}}{\operatorname{Pr}}[\mathcal{E}]
$$

to prove Theorem 23 it suffices to bound the expected value conditioned on event $\mathcal{E}$ by $\Omega\left(\frac{\log m}{\log \log m}\right)$. Therefore, in the remainder of this section we assume that event $\mathcal{E}$ happens.

Lemma 28. The loads of the machines within the same class differ only slightly. In particular, $\left|L_{i}-L_{i^{\prime}}\right| \leq 1 / 8$ for any machines $i, i^{\prime} \in M_{h}$.

Proof. Suppose to the contrary that there exist two machines $i, i^{\prime} \in M_{h}$ such that $L_{i}-L_{i^{\prime}}>$ $1 / 8$. Recall that according to the LPT rule all type $A$ jobs will be assigned to the machines before the type $B$ jobs are assigned. After all type $A$ jobs have been assigned to the machines in $M_{h}$, the difference in load between any two machines in $M_{h}$ is at most 1 since $p_{j} \leq 1$ for all jobs $j$.

Since the processing time of all type $B$ jobs is bounded by $1 / 8, L_{i}-L_{i^{\prime}}>1 / 8$ implies that no type $B$ job is assigned to machine $i$ nor to any machine that has load at least $L_{i}$. Hence, all type $B$ jobs are assigned to the machines that have load less than $L_{i}$. Note that there are at most $m_{h}-1$ such machines.

As the difference in load between machine $i$ and any other machine in $M_{h}$ is at most 1 , the total amount of processing requirements of type $B$ jobs in class $M_{h}$ is bounded by $Q_{h}^{B} \leq\left(m_{h}-1\right) \cdot 1<17 m_{h} / 16-m_{h} / 32=\mathbf{E}\left[Q_{h}^{B}\right]-m_{h} / 32$ contradicting the assumption that event $\mathcal{E}_{h}^{B}$ holds.

Lemma 29. For any machine $i \in M_{h}$ the inequality

$$
\left|L_{i}-\frac{1}{m_{h}}\left(\mathbf{E}\left[Q_{h}^{A}\right]+\mathbf{E}\left[Q_{h}^{B}\right]\right)\right| \leq \frac{7}{32}
$$

holds, i.e., the load of machine $i$ is close to the expected average machine load in class $M_{h}$. 
Proof. By applying the triangle inequality we obtain

$$
\begin{aligned}
\left|L_{i}-\frac{\mathbf{E}\left[Q_{h}^{A}\right]+\mathbf{E}\left[Q_{h}^{B}\right]}{m_{h}}\right| & \leq\left|L_{i}-\frac{Q_{h}^{A}+Q_{h}^{B}}{m_{h}}\right|+\frac{\left|Q_{h}^{A}-\mathbf{E}\left[Q_{h}^{A}\right]\right|}{m_{h}}+\frac{\left|Q_{h}^{B}-\mathbf{E}\left[Q_{h}^{B}\right]\right|}{m_{h}} \\
& \leq\left|L_{i}-\frac{\sum_{i^{\prime} \in M_{h}} L_{i^{\prime}}}{\left|M_{h}\right|}\right|+\frac{1}{16}+\frac{1}{32} \leq \frac{7}{32},
\end{aligned}
$$

where the second inequality holds since $\mathcal{E}_{h}^{A}$ and $\mathcal{E}_{h}^{B}$ are true. The last inequality is due to Lemma 28.

Lemma 30. Schedule $\sigma$ is lex-jump optimal.

Proof. We need to show that $L_{i^{\prime}}+p_{j} \geq L_{i}$ holds for any machine $i \in M_{h}$, any job $j \in J_{i}$, and any machine $i^{\prime} \in \mathcal{M}_{j}$. Let $i \in M_{h}$ be an arbitrary machine. First, consider the last job $j$ that has been assigned to $i$. Then, $L_{i^{\prime}}+p_{j} \geq L_{i}$ for any machine $i^{\prime} \in M_{h}$ as this job was assigned to machine $i$ by list scheduling. Furthermore, job $j$ is a smallest job on machine $i$ due to the LPT rule. Hence, $L_{i^{\prime}}+p_{j^{\prime}} \geq L_{i}$ for any machine $i^{\prime} \in M_{h}$ and any job $j^{\prime} \in J_{i}$ assigned to machine $i$.

For type $B$ jobs on machine $i$ the set of allowed machines equals $M_{h}$. It just remains to show that $L_{i^{\prime}}+p_{j} \geq L_{i}$ for any machine $i^{\prime} \in M_{h+1}$ and any type $A$ job $j \in J_{i}$ with $i \in M_{h}$. Recalling $\left.a_{h}=\left\lceil\left(a_{h-1} / a_{h-2}\right)-7 / 15\right) \cdot a_{h-1}\right\rceil$ for $h \geq 2, m_{h}=a_{h-1}$, and $b_{h} / m_{h}=17$ we observe that

$$
\begin{aligned}
\frac{\mathbf{E}\left[Q_{h+1}^{A}\right]+\mathbf{E}\left[Q_{h+1}^{B}\right]}{m_{h+1}} & =\frac{\frac{15}{16} a_{h+1}+\frac{1}{16} b_{h+1}}{m_{h+1}}=\frac{15}{16} \cdot \frac{a_{h+1}}{a_{h}}+\frac{1}{16} \cdot \frac{b_{h+1}}{m_{h+1}} \\
& \geq \frac{15}{16} \cdot\left(\frac{a_{h}}{a_{h-1}}-\frac{7}{15}\right)+\frac{1}{16} \cdot \frac{b_{h}}{m_{h}} \\
& =\frac{\mathbf{E}\left[Q_{h}^{A}\right]+\mathbf{E}\left[Q_{h}^{B}\right]}{m_{h}}-\frac{7}{16}
\end{aligned}
$$

for any $h=1, \ldots, z_{k}-1$. This implies

$$
\begin{aligned}
L_{i^{\prime}}+p_{j} & \geq \frac{\mathbf{E}\left[Q_{h+1}^{A}\right]+\mathbf{E}\left[Q_{h+1}^{B}\right]}{m_{h+1}}-\frac{7}{32}+\frac{7}{8} \\
& \geq \frac{\mathbf{E}\left[Q_{h}^{A}\right]+\mathbf{E}\left[Q_{h}^{B}\right]}{m_{h}}-\frac{7}{16}+\frac{21}{32} \\
& =\frac{\mathbf{E}\left[Q_{h}^{A}\right]+\mathbf{E}\left[Q_{h}^{B}\right]}{m_{h}}+\frac{7}{32} \geq L_{i},
\end{aligned}
$$

where the first and the last inequality are due to Lemma 29 .

Finally, we can prove Theorem 23 . 
Proof of Theorem 23. As mentioned before, due to Lemma 27 it suffices to bound the expected value conditioned on event $\mathcal{E}$. If event $\mathcal{E}$ holds, then schedule $\sigma=\sigma(I)$ is lexjump optimal (see Lemma 30), i.e., $\sigma \in \operatorname{Lex}(I)$, and has makespan

$$
\begin{aligned}
C_{\max } & \geq \max _{i \in M_{1}} L_{i} \geq \frac{Q_{1}^{A}+Q_{1}^{B}}{m_{1}} \geq \frac{\mathbf{E}\left[Q_{1}^{A}\right]+\mathbf{E}\left[Q_{1}^{B}\right]}{m_{1}}-\frac{\frac{m_{1}}{16}+\frac{m_{1}}{32}}{m_{1}} \\
& =\frac{\frac{15}{16} k^{3}+\frac{1}{16} \cdot 17 k^{2}}{k^{2}}-\frac{3}{32} \geq \frac{15}{16} k,
\end{aligned}
$$

where the third inequality is due to the occurrence of $\mathcal{E}_{1}^{A}$ and $\mathcal{E}_{1}^{B}$. Now, consider the following schedule $\sigma^{\prime}$ :

- For $h=1, \ldots, z_{k}-1$ spread the jobs of class $\mathcal{J}_{h}^{A}$ evenly among the machines in class $M_{h+1}$. As $\left|\mathcal{J}_{h}^{A}\right|=a_{h}=m_{h+1}=\left|M_{h+1}\right|$, each machine is assigned exactly one type $A$ job.

- Spread the jobs of class $\mathcal{J}_{z_{k}}^{A}$ evenly among the machines in class $M_{z_{k}}$. As $\left|\mathcal{J}_{z_{k}}^{A}\right|=$ $a_{z_{k}} \leq a_{z_{k}-1}=m_{z_{k}}=\left|M_{z_{k}}\right|$, each machine is assigned at most one type $A$ job.

- For $h=1, \ldots, z_{k}$ spread the jobs of class $\mathcal{J}_{h}^{B}$ evenly among the machines in class $M_{h}$. As $\left|\mathcal{J}_{h}^{B}\right|=17 m_{h}=17 \cdot\left|M_{h}\right|$, each machine is assigned exactly 17 type $B$ jobs.

Note that with 'evenly' we refer to the number of jobs on each machine and not to the load. Figure 9 shows schedule $\sigma^{\prime}$ where each machine $h$ is a representative for all machines in class $M_{h}$.

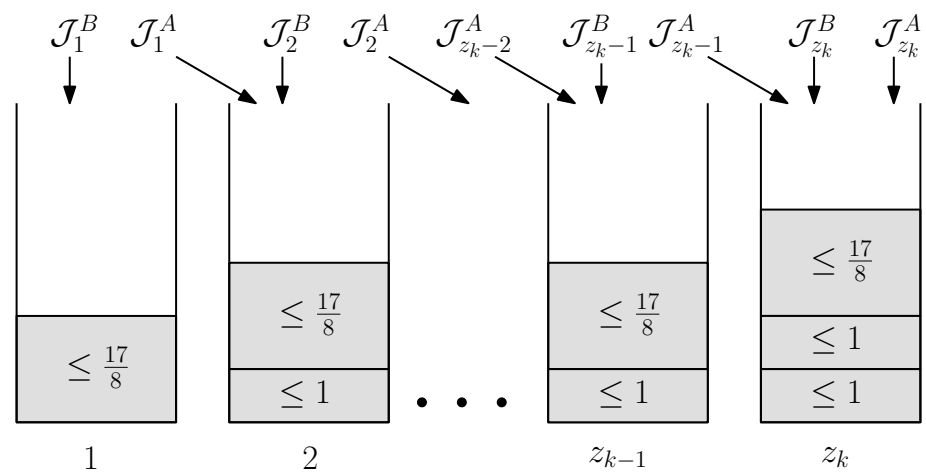

Figure 9: Schedule $\sigma^{\prime}$

As each machine contains at most 2 type $A$ jobs and 17 type $B$ jobs, the makespan of schedule $\sigma^{\prime}$ and hence $C_{\max }^{*}$ is bounded by $2 \cdot 1+17 \cdot 1 / 8 \leq 5$. This implies $C_{\max }(\sigma) / C_{\max }^{*} \geq$ $3 k / 16=\Omega\left(\Gamma^{-1}(m)\right)$ due to Lemma 26 . Hence,

$$
\underset{I \sim \mathcal{I}}{\mathbf{E}}\left[\max _{\sigma \in \operatorname{Lex}(I)} \frac{C_{\max }(I, \sigma)}{C_{\max }^{*}(I)} \mid \mathcal{E}\right] \geq \underset{I \sim \mathcal{I}}{\mathbf{E}}\left[\frac{C_{\max }(I, \sigma(I))}{C_{\max }^{*}(I)} \mid \mathcal{E}\right]=\Omega\left(\frac{\log m}{\log \log m}\right) .
$$

Remark. The worst case upper bound on the performance guarantee for lex-jump optimal schedules on restricted related machines is $O\left(\frac{\log S}{\log \log S}\right)$, where $S=\sum_{i} s_{i} / s_{m}$ [21]. As for 
identical machines $S=m$, i.e., each machine has speed 1, the upper bound matches the lower bound of Theorem 23 up to a constant factor and smoothing does also not improve the performance guarantee for the worst lex-jump optimal schedules on restricted related machines.

Lemma 27 established that $\mathcal{E}$ occurs with high probability. Hence, if we choose $k$ suitably large, the stated results not only hold in expectation, but also with high probability.

\section{Concluding Remarks}

We have proven that the lower bounds for all scheduling variants with restricted machines are rather robust against random noise, not only in expectation but even with high probability. We have also shown that the situation looks much better for unrestricted machines where we obtained performance guarantees of $\Theta(\phi)$ and $\Theta(\log \phi)$ for the jump and lex-jump algorithm, respectively. The latter bound also holds for the price of anarchy of routing on parallel links and for the list scheduling algorithm, even when the order in which the jobs are presented to the algorithm can be chosen by the adversary when the realization of the processing times are known.

There are several interesting directions of research and we view our results only as a first step towards fully understanding local search and greedy algorithms in the framework of smoothed analysis. For example, we have only perturbed the processing requirements, and it might be the case that the worst-case bounds for the restricted scheduling variants break down if also the sets $\mathcal{M}_{j}$ are to some degree random. In general it would be interesting to study different perturbation models where the sets $\mathcal{M}_{j}$ and/or the speeds $s_{i}$ are perturbed. Lemma 15 and Corollary 17 indicate that there need to exist many machines having exponentially small speeds. We conjecture that if speeds are being smoothed, then the smoothed performance guarantee of near list schedules on restricted related machines is $\Theta(\log \phi)$ as well.

Another interesting question is the following: since we do not know which local optimum is reached, we have always looked at the worst local optimum. It might, however, be the case that the local optima reached in practice are better than the worst local optimum. It would be interesting to study the quality of the local optimum reached under some reasonable assumptions on how exactly the local search algorithms work. An extension in this direction would be to analyze the quality of coordination mechanisms under smoothing.

\section{Acknowledgments}

We thank three anonymous referees for their valuable comments and suggestions that helped to improve the writing of the paper.

\section{References}

[1] E. Angel. A survey of approximation results for local search algorithms. In E. Bampis, K. Jansen, and C. Kenyon, editors, Efficient Approximation and Online Algorithms, 
volume 3484 of LNCS, pages 30-73. Springer-Verlag, Heidelberg, Germany, 2006.

[2] J. Aspnes, Y. Azar, A. Fiat, S. A. Plotkin, and O. Waarts. On-line routing of virtual circuits with applications to load balancing and machine scheduling. Journal of the ACM, 44(3):486-504, 1997.

[3] B. Awerbuch, Y. Azar, Y. Richter, and D. Tsur. Tradeoffs in worst-case equilibria. Theoretical Computer Science, 361:200-209, 2006.

[4] L. Becchetti, S. Leonardi, A. Marchetti-Spaccamela, G. Schäfer, and T. Vredeveld. Average case and smoothed competitive analysis for the multi-level feedback algorithm. Mathematics of Operations Research, 31(3):85-108, 2006.

[5] R. Beier and B. Vöcking. Random knapsack in expected polynomial time. Journal of Computer and System Sciences, 69(3):306-329, 2004.

[6] Y. Cho and S. Sahni. Bounds for list schedules on uniform processors. SIAM Journal on Computing, 9:91-103, 1980.

[7] A. Czumaj and B. Vöcking. Tight bounds for worst-case equilibria. Transactions on Algorithms ACM, 3(1), 2007.

[8] M. Englert, H. Röglin, and B. Vöcking. Worst case and probabilistic analysis of the 2-opt algorithm for the TSP. In Proceedings of the 18th ACM-SIAM Symposium on Discrete Algorithms (SODA), pages 1295-13004, 2007.

[9] G. Finn and E. Horowitz. A linear time approximation algorithm for multiprocessor scheduling. BIT, 19:312-320, 1979.

[10] M. R. Garey and D. S. Johnson. Computers and Intractibility: A Guide to the Theory of NP-Completeness. W.H. Freeman \& Co., New York, NY, 1979.

[11] C. A. Glass and H. Kellerer. Parallel machine scheduling with job assignment restrictions. Naval Research Logistics, 54(3):250-257, 2007.

[12] R. L. Graham. Bounds for certain multiprocessing anomalies. Bell System Technical Journal, 45:1563-1581, 1966.

[13] R. L. Graham, E. L. Lawler, J. K. Lenstra, and A. H. G. Rinnooy Kan. Optimization and approximation in deterministic sequencing and scheduling: a survey. Annals of Discrete Mathematics, 5:287-326, 1979.

[14] D. S. Hochbaum and D. B. Shmoys. A polynomial approximation scheme for machine scheduling on uniform processors: using the dual approximation approach. SIAM Journal on Computing, 17:539-551, 1988.

[15] M. Hoefer and A. Souza. Tradeoffs and average-case equilibria in selfish routing. ACM Transactions on Computation Theory, 2(1):article 2, 2010.

[16] W. Hoeffding. Probability inequalities for sums of bounded random variables. Journal of the American Statistical Association, 58(301):13-30, 1963. 
[17] J. Y. T. Leung and C. L. Li. Scheduling with processing set restrictions: A survey. International Journal of Production Economics, 116:251-262, 2008.

[18] C. L. Li. Scheduling unit-length jobs with machine eligibility restrictions. European Journal of Operational Research, 174:1325-1328, 2006.

[19] W. P. A. J. Michiels, E. H. L. Aarts, and J. H. M. Korst. Theoretical Aspects of Local Search. Springer-Verlag, Heidelberg, Germany, 2007.

[20] J. Ou, J. Y.-T. Leung, and C. L. Li. Scheduling parallel machines with inclusive set restrictions. Naval Research Logistics, 55(4):328-338, 2008.

[21] C. Rutten, D. Recalde, P. Schuurman, and T. Vredeveld. Performance guarantees of jump neighborhoods on restricted related parallel machines. Operations Research Letters, 40:287-291, 2012.

[22] G. Schäfer and N. Sivadasan. Topology matters: Smoothed competitiveness of metrical task systems. Theoretical Computer Science, 341(1-3):3-14, 2005.

[23] P. Schuurman and T. Vredeveld. Performance guarantees of local search for multiprocessor scheduling. Informs Journal on Computing, 19(1):52-63, 2007.

[24] D. A. Spielman and S. H. Teng. Smoothed analysis of algorithms: Why the simplex algorithm usually takes polynomial time. Journal of the ACM, 51(3):385-463, 2004.

[25] D. A. Spielman and S. H. Teng. Smoothed analysis: an attempt to explain the behavior of algorithms in practice. Communications of the ACM, 52(10):76-84, 2009.

[26] B. Vöcking. Selfish load balancing. In N. Nisan, T. Roughgarden, E. Tardos, and V. Vazirani, editors, Algorithmic Game Theory, chapter 20. Cambridge University Press, New York, NY, USA, 2007. 


\section{A Table of notation}

In the table below, the notation used in this paper is summarized.

\begin{tabular}{|ll|}
\hline$J$ & set of jobs $1, \ldots, n$ \\
$M$ & set of machines $1, \ldots, m$ \\
$p_{j}$ & processing requirement of job $j$ \\
$s_{i}$ & speed of machine $i$ \\
$\mathcal{M}_{j}$ & set of machines on which job $j$ can be scheduled \\
$s_{\max }$ & maximum speed of the machines \\
$s_{\min }=1$ & minimum speed of the machines; \\
& by scaling we assume w.l.o.g. it to be 1. \\
$C_{\max }^{*}$ & optimal makespan \\
$C_{\max }(\sigma)$ & makespan of schedule $\sigma$ \\
$J_{i}(\sigma)$ & set of jobs scheduling on machine $i$ in schedule $\sigma$ \\
$L_{i}(\sigma)$ & $=\sum_{j \in J_{i}(\sigma)} p_{j} / s_{i}$ \\
& load of machine $i$ in schedule $\sigma$. \\
$J_{i, j}(\sigma)$ & $=J_{i}(\sigma) \cap\{1, \ldots, j\}$ \\
$j_{i}^{t}$ & $=\min \left\{j: \sum_{\ell \in J_{i, j}}(\sigma) p_{\ell} / s_{i} \geq t \cdot C_{\max }^{*}\right\}$ \\
$J_{i, \geq t}(\sigma)$ & $=J_{i, t_{i}^{t}}(\sigma)$ \\
$c$ & $=\left\lfloor\frac{C_{\max }(\sigma)}{C_{\max }^{*}}\right\rfloor-1$ \\
$i_{k}$ & $=\max \left\{i \in M: L_{i^{\prime}} \geq k \cdot C_{\max }^{*} \forall i^{\prime} \leq i\right\}$ \\
$H_{k}$ & $=\left\{1, \ldots, i_{k}\right\}$ \\
$R_{k}$ & $=H_{k} \backslash H_{k+1}$ for $k=0,1, \ldots, c-1$ \\
$R_{c}$ & $=H_{c}$. \\
\end{tabular}

\section{B Hoeffding's bound}

On several occasions in this paper we use Hoeffding's bound [16] to bound tail probabilities. For completeness, we state the bound in the following theorem.

Theorem 31. Let $X_{1}, \ldots, X_{n}$ be independent random variables. Define $X:=\sum_{j=1}^{n} X_{j}$ and $\mu=\mathbf{E}[X]$. If each $X_{j} \in\left[a_{j}, b_{j}\right]$ for some constants $a_{j}$ and $b_{j}, j=1, \ldots, n$, then for any $t>0$

$$
\begin{aligned}
& \operatorname{Pr}[X \leq \mathbf{E}[X]-t] \leq \exp \left(\frac{-2 t}{\sum_{j}\left(b_{j}-a_{j}\right)^{2}}\right), \quad \text { and }, \\
& \operatorname{Pr}[X \geq \mathbf{E}[X]+t] \leq \exp \left(\frac{-2 t}{\sum_{j}\left(b_{j}-a_{j}\right)^{2}}\right) .
\end{aligned}
$$

